\title{
Universality in the Spatial Evolution of Self-Aggregation of Tropical Convection $\mathscr{\mathscr { C }}$
}

\author{
Julia M. WindmilleR ${ }^{\mathrm{a}}$ AND GeORGE C. CRAig \\ Ludwig-Maximilians-Universität München, Munich, Germany
}

(Manuscript received 27 April 2018, in final form 21 March 2019)

\begin{abstract}
Self-aggregation in numerical simulations of tropical convection is described by an upscale growth and intensification of dry and moist regions. Previous work has focused on determining the relevant mechanism that induces moist regions to get moister and dry regions to get drier. Though different mechanisms have been identified, the spatial evolution of self-aggregation is remarkably universal. The first part of this study shows that different mechanisms can lead to a similar evolution of self-aggregation, if self-aggregation is described by a phase separation of moist and dry regions, through a process called coarsening. Though it was previously introduced based on a convection-humidity feedback, coarsening, importantly, is not tied to a specific feedback process but only requires an intensification of local humidity perturbations. Based on different feedback loops, three simple models of the evolution of the humidity field are introduced, all of which lead to coarsening. In each model, diffusive transport of humidity is assumed, which approximates a humidity increase due to convection, within a finite region around convective cores. In the second part, predictions made by coarsening are compared with atmospheric model simulations. Analyzing a set of radiative-convective equilibrium simulations shows that coarsening correctly predicts the upscale growth of the moist and dry regions in the early stages of self-aggregation. In addition, coarsening can explain why self-aggregation is not observed for small domains and why the shape of the final moist region changes with the shape of the domain.
\end{abstract}

\section{Introduction}

\section{a. Self-aggregation of tropical convection}

Organization of tropical convection has received increased attention in recent years. One key question, as outlined by Bony et al. (2015), is to determine the role of convective organization for climate. However, one difficulty when discussing convective organization is to define what we mean by organization. One possible definition has been given by Mapes and Neale (2011), who state that convective organization is the "nonrandomness in meteorological fields in convecting regions." This definition includes a large number of different

\footnotetext{
T) Supplemental information related to this paper is available at the Journals Online website: https://doi.org/10.1175/JAS-D-180129.s1.

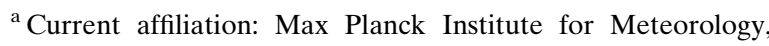
Hamburg, Germany.
}

Corresponding author: J. M. Windmiller, julia.windmiller@ mpimet.mpg.de phenomena, ranging from the organization of shallow cumulus clouds at the boundaries of cold pools (e.g., Seifert and Heus 2013) over mesoscale convective clusters (e.g., Houze 2004) to large-scale anomalies in the vertically integrated humidity field that impact the spatial distribution of deep convection (e.g., Mapes et al. 2018).

The importance of tropospheric humidity on deep convection has been investigated in a number of studies. Deep convection, if measured by precipitation rate, has been shown to increase nonlinearly with column relative humidity (e.g., Bretherton et al. 2004, 2005). Some studies have argued that this relationship is a result of convection to self-organize with respect to humidity (e.g., Peters and Neelin 2006; Yano et al. 2012). While in the context of self-organization, convection is expected to drive the tropospheric humidity content toward a critical value (Peters and Neelin 2006), numerical simulations of radiative-convective equilibrium (RCE) have shown that humidity anomalies can form in atmospheric models, even in the presence of homogeneous boundary and initial conditions. This is not a contradiction to the findings of Peters and Neelin (2006) as convection, which is limited to the moist regions, might still drive the humidity content of the moist region toward a critical value but suggests that 
additional processes induce the separation of the domain into moist and dry regions and thus the separation of the domain into regions with and without deep convection.

This spontaneous spatial organization of convection in RCE, despite homogeneous boundary conditions and forcing, has been defined as self-aggregation (SA; Wing et al. 2017). The organized state is referred to as being aggregated as it is marked by positive spatial and temporal correlations between deep convective updrafts. In particular, the domain is divided into distinct regions: dry regions without convection and moist regions with convection. These regions grow upscale in time, often until a single moist and convecting region is surrounded by a dry and subsiding region. As this evolution arises in the absence of heterogeneous boundary conditions or forcing it is referred to as self-aggregation. Note that the term aggregation in this context is slightly misleading as positive correlations between convective updrafts are not necessarily due to merging of individual convective updrafts into a single convective cluster but can also be due to the growth of dry regions where there is no convection.

Self-aggregation of convection was first observed by Held et al. (1993), who showed the transition from randomly distributed to aggregated convection in a 2D RCE simulation. Since then, self-aggregation has been observed in a large number of RCE simulations (Tompkins 2001; Posselt et al. 2012; Wing and Cronin 2016; Yang 2018a,b; Tompkins and Craig 1998; Bretherton et al. 2005; Muller and Held 2012; Kempf 2013; Jeevanjee and Romps 2013; Wing and Emanuel 2014; Muller and Bony 2015; Holloway and Woolnough 2016; Hohenegger and Stevens 2016; Popke et al. 2013; Coppin and Bony 2015; Reed et al. 2015; Becker et al. 2017; Arnold and Putman 2018). The setup of the simulation impacts self-aggregation. In particular, SA does not occur at all if the domain size is too small (e.g., Bretherton et al. 2005; Muller and Held 2012) and does not start spontaneously for resolutions higher than about $2 \mathrm{~km}$ but persists when started from aggregated initial conditions (Muller and Held 2012). In addition, the appearance of self-aggregation depends on the domain shape. In channel-like simulations the moist regions always appear as bands (Tompkins 2001; Posselt et al. 2012; Wing and Cronin 2016) and though some studies have shown that band-like structures can (at least transiently) also form in square domains (Tompkins and Craig 1998; Wing and Emanuel 2014; Muller and Bony 2015), moist regions in square domains generally tend toward a single circular region (e.g., Bretherton et al. 2005; Muller and Held 2012; Kempf 2013; Jeevanjee and Romps 2013; Hohenegger and Stevens 2016).

The above listed studies specifically refer to selfaggregation under conditions of nonrotating RCE. While we will focus on self-aggregation in the nonrotating setup in this study, it should be noted that rotation significantly affects self-aggregation. In fact, as summarized by Yang (2018b), the self-aggregation clusters in simulations with the Coriolis effect resemble tropical cyclones [for a review, see Wing et al. (2017)] while a propagating selfaggregation cluster that has been found in a simulation that includes the beta effect has been noted to broadly resemble the Madden-Julian oscillation (Yano et al. 2012).

As reviewed by Wing et al. (2017), different mechanisms have been proposed to drive self-aggregation. ${ }^{1}$ While we will discuss three of these mechanisms in detail in section 2, a short overview of the relevant processes is given in the following. Though it is difficult to determine which mechanism drives self-aggregation in a specific setup, a number of relevant processes have been identified. These processes are surface fluxes, radiation (longwave and shortwave), virtual effect of water vapor, and convective entrainment and detrainment. Using a diagnostic introduced by Wing and Emanuel (2014), one can, for a specific simulation, determine for each process whether it leads to an amplification or a suppression of humidity anomalies. In the former case, we say that the process results in a positive feedback, favoring selfaggregation, while in the latter case, we say it leads to a negative feedback, opposing self-aggregation. There is evidence that there is no unique process relevant for SA. Studies have shown that the amplification of humidity perturbations is generally due to more than one process and that the relevant processes change with sea surface temperature (SST; Wing and Cronin 2016; Coppin and Bony 2015), with the presence or absence of cold pools (Muller and Bony 2015; Holloway and Woolnough 2016), and during different stages of SA (Wing and Emanuel 2014; Wing and Cronin 2016; Coppin and Bony 2015; Holloway and Woolnough 2016; Yang 2018a). Note that, because of the strong positive correlation between humidity and convection, SA is often, and will be in this study, investigated in terms of the smoother humidity field.

A change in the relevant process also indicates that the mechanism driving SA changes. While one might assume that the appearance of SA depends on the mechanism that drives it, the spatial evolution of SA is surprisingly universal, being generally described by an upscale growth of moist and dry regions, instead of, for example, the intensification of a preferred wavelength. This suggests that the type of pattern formation in SA is similar in all studies and does not depend sensitively on the relevant mechanism. In contrast to the mechanism

\footnotetext{
${ }^{1}$ Note that a further mechanism involving the virtual effect of water vapor was proposed by Yang (2018a).
} 
that drives SA, the spatial evolution of SA has received little attention though two studies have investigated the spatial scales in the steady state (Wing and Cronin 2016; Yang 2018b).

To understand the spatial evolution of the humidity field during SA, one has to assess not only how perturbations in the (vertically integrated) humidity field amplify but also how humidity is exchanged between different regions. Considering the mechanism driving SA with respect to this exchange in humidity, two categories are naturally distinguished:

- Advective mechanisms: Humidity perturbations amplify as moisture is advected out of dry into moist regions, for example, because of a circulation driven by differential radiative cooling.

- Local mechanisms: Humidity perturbations amplify as the moisture content of moist (dry) columns increases (decreases), for example, by increased (decreased) surface fluxes.

Analysis of the budget equation of the variability of the moist static energy (Wing and Emanuel 2014; Holloway and Woolnough 2016) suggests that local processes are most important for SA, at least during the onset of SA. However, if local processes are relevant, they contain no information about how humidity is exchanged within the domain, and connected dry and moist regions can only emerge if such an exchange exists. To describe the spatial evolution during the onset of SA, we therefore need to introduce an additional term that accounts for moisture exchange with neighboring grid cells in addition to specifying a local mechanism.

In this study, we propose that the exchange of humidity, at least during the early stages of SA, is diffusive. We show that diffusion approximates the effect of moisture increase due to stochastically triggered convection. As diffusion leads to a downgradient moisture transport, this choice is in agreement with the initially negative contribution of the convergence term found by Wing and Emanuel (2014) although not with the neutral or even positive convergence term observed by Holloway and Woolnough (2016). We also note that while Coppin and Bony (2015) find that the overall advective tendency is negative, they show that there is still export of humidity out of the dry regions, suggesting a more complicated humidity exchange than pure diffusion. Although it is not the main focus of this paper, some evidence for a transition from a diffusion-dominated regime to an advectiondominated regime will be noted in section 5 below.

\section{b. Coarsening}

Assuming that the humidity content in the atmosphere is governed by local processes that induce moist

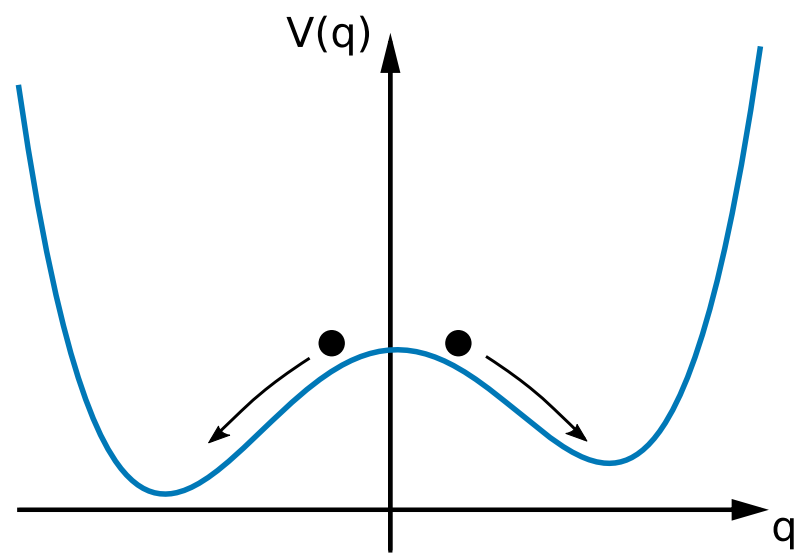

FIG. 1. Double-well potential representing bistability of $q$. Depending on whether $q$ is smaller or larger than the local maximum it evolves toward one or the other potential minimum.

regions to get moister and dry regions to get drier, the atmosphere is an example of a bistable system as each location tends toward one of two phases (moist or dry), depending on its initial condition. In particular, expressing the evolution of the humidity content $q$ in terms of a potential $V(q)$,

$$
\frac{\partial q}{\partial t}=-\frac{\delta V}{\delta q}
$$

bistability results from a potential with double-well structure, with two (local) minima separated from each other by a local maximum. The state of $q$ corresponding to the local maximum is unstable as, when slightly perturbed, $q$ evolves toward one or the other potential minimum (see Fig. 1). In contrast, the two states corresponding to the two potential minima are stable to small perturbations. The explicit form of the potential $V(q)$ is determined by the physical processes driving the evolution of $q$, with the gradient of the potential (and thus the rate of evolution) at a given $q$ determined by the strength of the acting processes. Note that the evolution of the state away from the unstable maximum is sometimes referred to as a feedback process since the gradient of the potential increases away from the maximum, accelerating the rate of change of $q$.

Considering a spatially extended bistable system that allows for some mixing between regions of different phases, the regions will grow in size over time, through a process called coarsening (e.g., Bray 1994; Krapivsky et al. 2010). A simple mathematical model of coarsening is based on the following two assumptions (see, e.g., Krapivsky et al. 2010). The first assumption is that the thermodynamics of the bistable system is given by the Landau free energy: 


$$
F[q(\mathbf{x})]=\int\left\{V[q(\mathbf{x})]+\frac{D}{2}[\nabla q(\mathbf{x})]^{2}\right\} d \mathbf{x},
$$

where $V(q)$ is again a potential with double-well structure and $D$ is a diffusion coefficient. The second assumption is that the evolution of $q(\mathbf{x})$ is given by

$$
\frac{\partial q(\mathbf{x})}{\partial t}=-\frac{\delta F}{\delta q}=-\frac{\delta V}{\delta q}+D \nabla^{2} q(\mathbf{x})
$$

that is, tending toward minimizing $F(q)$ at each location $\mathbf{x}$. This equation is usually referred to as the timedependent Ginzburg-Landau equation ${ }^{2}$ (TDGL). Note that the time evolution described by the TDGL only leads to coarsening, that is, to an upscale growth of regions of the two phases, when started from a slightly perturbed field in the unstable state. Or, expressed differently, coarsening is the process that results from the time evolution of the TDGL for these specific initial conditions.

In Eqs. (2) and (3), the first term on the right-hand side represents the local bistability of the system in terms of the double-well potential and the second term includes the effects of horizontal mixing by diffusion. Note that the Landau free energy as well as the TDGL cannot be derived from first principles but are motivated by the coarsening found for the Ising model, a simple model for ferromagnets. Details on the theoretical background of coarsening can be found in the respective chapter of Krapivsky et al. (2010).

In the following, we will briefly review the evolution expected for $q(\mathbf{x})$ resulting from the TDGL, that is, Eq. (3). This discussion is simplified by noting that the evolution of the TDGL minimizes the Landau free energy of the system, that is, $d F / d t \leq 0$, as can be readily shown from Eqs. (2) and (3). Starting from a slightly perturbed field in the unstable state will first lead to the formation of small, coherent regions of the two stable states, which minimizes the contribution from the first term in Eq. (2), as the two stable states correspond to the two potential minima. At the boundaries of these regions, $q$ smoothly transitions from the $q$ value corresponding to the one potential minimum to the $q$ value of the other minimum. The smooth transition between the two regions, usually referred to as domain wall, can be understood as a trade-off between the first term in Eq. (2), which favors a sharp gradient between the different stable regions, and the second term, which favors weak gradients. The properties of the domain

\footnotetext{
${ }^{2}$ Note that the TDGL is sometimes also referred to as AllenCahn equation.
}

walls are entirely determined by the potential and the diffusion coefficient and thus independent of domain size, which will become important below. With time, the stable regions grow upscale, which reduces the total length of the boundary and thus the contribution of the domain walls to the Landau free energy.

Note that for minimizing Eq. (2), it is also important to distinguish whether the potential minima are degenerate, that is, the depths of both wells in $V(q)$ are identical, or nondegenerate, that is, the depths of the two wells are different. In the latter case, the regions of $q$ corresponding to the less deep potential well are only metastable as $F[q(\mathbf{x})]$ is further minimized by the stable regions ( $q$ corresponding to the deeper potential well) expanding into the metastable regions. In this case, the final state, that is, the state of minimal $F[q(\mathbf{x})]$, is reached when the entire domain is in the stable state. In the degenerate case, the final state is reached when the entire domain is in one of the two stable states.

While the precise time evolution depends on the potential function, some properties of the evolution are independent of the details of the potential. These properties are referred to as universal. In particular, the key property of coarsening is that the upscale growth of the stable regions is dynamically self-similar, with the typical length scale of the stable regions proportional to time raised to some power, here, $t^{1 / 2}$. In the remainder of the text, we will refer to this property as $t^{1 / 2}$ scaling. Note that dynamical exponent of $1 / 2$ is universal as long as $q$ is not a conserved quantity and the dimension of the system is larger than one.

As it will become important in the following discussion, we finally note that the TDGL is a specific version of a reaction-diffusion equation:

$$
\frac{\partial q}{\partial t}=R(q)+D \nabla^{2} q
$$

As in the TDGL equation, the change in $q$ in a reactiondiffusion equation depends on local processes (described by $R$ ) and diffusive mixing. The important difference to the TDGL is that, in a reaction-diffusion equation, $R$ can take any form and is not necessarily given by the functional derivative of a double-well potential $V(q)$.

Coarsening described by the TDGL was first proposed to describe SA by Craig and Mack (2013). Introducing a model that quantifies the positive feedback between convection and humidity based on convective entrainment and detrainment and assuming a diffusive exchange of humidity, they showed that the resulting model led to upscale growth of moist and dry regions comparable to SA. Their model has, however, two limitations. First, analysis of simulations showing self-aggregation suggest that the relevant feedback 
process might be different from the one proposed in Craig and Mack (2013), and second, their assumption of a diffusive interaction term lacks physical motivation.

In this paper, we further investigate the hypothesis that the upscale growth of moist and dry regions during SA is described by coarsening. In section 2, we consider the local bistability of the atmospheric humidity content. Comparing the time evolution equations for local humidity perturbations introduced by Bretherton et al. (2005), Craig and Mack (2013), and Emanuel et al. (2014) in section 2, we find that they are all unstable to small perturbations, though because of different mechanisms. These instabilities are then expressed in terms of a double-well potential, that is, as the time evolution due to the local process of the TDGL. In section 3, we investigate the horizontal mixing term and show that a diffusive spatial coupling term can result from a stochastic representation of convection. This motivates our choice of a diffusive transport term for the evolution equation of SA. Combining each of the three evolution equations with a diffusion term in section 4 leads to coarsening described by the TDGL in each case. Comparing the spatial evolution of the three models helps us illustrate the properties of coarsening that are independent of feedback details. In section 5, we demonstrate that the universal $t^{1 / 2}$ scaling of coarsening can explain the upscale growth of moist and dry regions and that coarsening in general can explain the dependence of SA on domain size and shape in RCE simulations.

\section{Three models based on local mechanisms}

In this paper, we suggest that the type of pattern formation observed during self-aggregation of convection is independent of the processes responsible for the positive feedback that drives self-aggregation, as long as the processes act locally. To illustrate this, we start by comparing the three theoretical models introduced by Bretherton et al. (2005), Craig and Mack (2013), and Emanuel et al. (2014), each quantifying processes that make the initially homogeneous humidity content unstable to perturbations. In particular, the models explain how humidity perturbations amplify with time, through a single process or a combination of processes, by introducing evolution equations for these perturbations. As we argue below, each evolution equation can be regarded as the local source term $R$ in a reactiondiffusion model.

In the following, each model, the relevant processes and the derivation method are summarized while a more extensive discussion is given in the online supplementary material. We note that the different models describe the time evolution of different humidity variables but that, for the purpose of this paper, we are principally interested in the distinction between whether the humidity is larger or smaller than the initial equilibrium content. We have therefore replaced the different humidity variables used in the different models by a generic scalar variable $q$, normalized to one. The original definition of $q$ for each model is given below with an overview of these definitions given in the appendix. In addition to this generalized humidity content $q$, we denote the mean humidity content in the absence of selfaggregation as $q_{\mathrm{RCE}}$ and the deviation from it as $q^{\prime}$.

\section{a. Model introduced by Bretherton et al. (2005)}

Bretherton et al. (2005) use a RCE simulation to derive a semiempirical model for the time evolution of the column relative humidity. Using the weak temperature gradient approximation, they derive an approximate budget equation for the column relative humidity from the budget equation of the vertically integrated frozen moist static energy. The resulting equation shows that column relative humidity mainly changes through radiation, surface fluxes, and moisture convergence. To obtain a closed equation for the time evolution of the column relative humidity, they proceed in two steps. As the first step, they determine how the radiation, surface flux, and moisture convergence components of the budget equation change in response to a change in precipitation by fitting their dependence on the perturbation precipitation rate $\left(P-P_{\mathrm{RCE}}\right)$. The change of the column relative humidity due to the three processes is then given by $\partial_{t} q=\left(P-P_{\mathrm{RCE}}\right) \sum_{i=1}^{3} f^{i}(q)$, where $f^{i}(q)$ refers to the empirically determined relation for process $i$. Note that they determine the dependence on precipitation rather than column relative humidity as, on physical grounds, they anticipate it to be the amount of convection that alters the forcing terms. As the second step, they use the dependence of the precipitation rate on humidity [their Eq. (2)] to express the precipitation rate $P$ in terms of humidity:

$$
P\left(q^{\prime}\right) \sim e^{k_{1}^{b} q^{\prime}} .
$$

As the resulting equation for $\partial_{t} q^{\prime}$ accounts for the local processes that modify a humidity perturbation but does not represent any humidity exchange between neighboring regions, we refer to it as a local source term $R_{B}$ [see Eq. (4)] in the following. ${ }^{3}$ As shown in the supplementary material, the equation derived by Bretherton et al. (2005) can be written as

\footnotetext{
${ }^{3}$ The local term actually includes the nonlocal convergence term but the diagnostic used to derive this term makes it impossible to include it as a transport term.
} 
TABLE 1. Parameters for positive feedback loop based on Bretherton et al. (2005).

\begin{tabular}{cccc}
\hline \hline & $k_{1}^{b}$ & $k_{2}^{b}$ & $k_{3}^{b}$ \\
\hline Value & 16.6 & $7.8 \times 10^{-8} \mathrm{~s}^{-1}$ & $1.3 \times 10^{-6} \mathrm{~s}^{-1}$ \\
\hline
\end{tabular}

$$
R_{B}\left(q^{\prime}\right)=\partial_{t} q^{\prime}=\left(e^{k_{1}^{b} q^{\prime}}-1\right)\left(k_{2}^{b}-k_{3}^{b} q^{\prime}\right),
$$

where $q^{\prime}$ denotes a perturbation of the column relative humidity with respect to the equilibrium content $q_{\mathrm{RCE}}$ and the parameters $k_{1}^{b}, k_{2}^{b}$, and $k_{3}^{b}$ combine multiple parameters given in Bretherton et al. (2005) to simplify the notation. Their values are calculated from the parameters given in Bretherton et al. (2005) and listed in Table 1.

\section{b. Model introduced by Craig and Mack (2013)}

The model introduced by Craig and Mack (2013) is based on a positive feedback between convection and the humidity content of the free troposphere. On the one hand, convection will increase the humidity in the free troposphere by transporting humidity out of the boundary layer. On the other hand, deep convection is more likely to occur in moist regions than in dry regions, as the entrainment of dry air reduces the buoyancy. They formalized this feedback on the humidity content as

$$
R_{C}(q)=-k_{1}^{c} q+k_{2}^{c}\left[\exp \left(k_{3}^{c} q\right)-1\right]\left(\frac{1}{k_{4}^{c} q}-1\right),
$$

where $q$ denotes the free-tropospheric column relative humidity and $k_{1}^{c}, k_{2}^{c}, k_{3}^{c}$, and $k_{4}^{c}$ are model parameters. Note that the source term $R_{C}$ again represents the evolution of the humidity content due to local processes.

The first term on the right-hand side of Eq. (7) represents the subsidence drying term, which accounts for the rate of change of humidity due to subsidence. The second term is the convective moistening term and represents the increase in humidity due to convection. The parameter values used here are summarized in Table 2, and a more detailed description of the individual terms together with the estimation of the parameters from a RCE simulation (Kempf 2014) is given in the appendix.

Craig and Mack (2013) also included a global constraint, which ensured that the domain can neither become completely dry nor completely moist, as is the case for a RCE simulation. As discussed in Craig and Mack (2013), the constraint mainly affects the final stage of selfaggregation, where it enforces the formation of a single circular moist region surrounded by a dry region. In the
TABLE 2. Parameters for positive feedback loop based on Craig and Mack (2013).

\begin{tabular}{ccccc}
\hline \hline & $k_{1}^{c}$ & $k_{2}^{c}$ & $k_{3}^{c}$ & $k_{4}^{c}$ \\
\hline Value & $2.0 \times 10^{-6} \mathrm{~s}^{-1}$ & $5.7 \times 10^{-9} \mathrm{~s}^{-1}$ & 11.4 & 1.1 \\
\hline
\end{tabular}

following discussion, we will focus on the early stages of self-aggregation and therefore neglect the constraint for better comparability with the other models.

\section{c. Model introduced by Emanuel et al. (2014)}

In their study, Emanuel et al. (2014) investigate the sea surface temperature dependence of self-aggregation. To this end, they first derive a time evolution equation for humidity perturbations by linearizing the budget equation of moist static energy around the radiativeconvective equilibrium state. Analyzing this budget equation and the results from Wing and Emanuel (2014), they argue that the SST dependence arises because of longwave radiation. Longwave radiation yields a positive feedback only for large SST, and the proposed feedback loop can be summarized as follows. In case of a moist lower troposphere, as expected for high SST, negative (positive) moisture perturbations lead to stronger (weaker) radiative cooling inducing mean descent (ascent) as horizontal temperature differences are removed by the compensating vertical motion in an atmosphere where the weak temperature approximation holds.

Here, we are only interested in conditions that lead to self-aggregation and therefore constrain our analysis of the model to the limit of high SST. As we show in the appendix, the initially two-layer model introduced by Emanuel et al. (2014) can then be represented by the following single-layer model:

$$
R_{E}\left(q^{\prime}\right)=k^{e} q^{\prime} \text { with } k^{e}=5.8 \times 10^{-6} \mathrm{~s}^{-1},
$$

where $q^{\prime}$ is the deviation from the mean RCE specific humidity in the upper free troposphere divided by the corresponding saturation value. The time evolution of $q^{\prime}$ is again referred to as a local source term $R_{E}$ and $k^{e}$ is a model parameter.

\section{d. Model comparison}

Each model introduced above describes how a perturbation in the humidity content $q^{\prime}$ evolves in time. While they are based on different processes, are derived in different ways, and even account for the humidity content of different layers, they have one important common property: humidity perturbations grow in time. This can be seen in Fig. 2a, where we show the local source term $R$ for each model, using the parameters 
a)

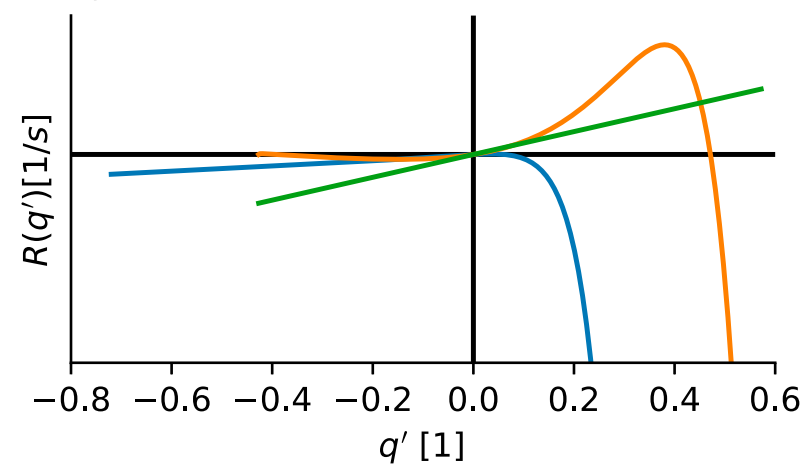

b)

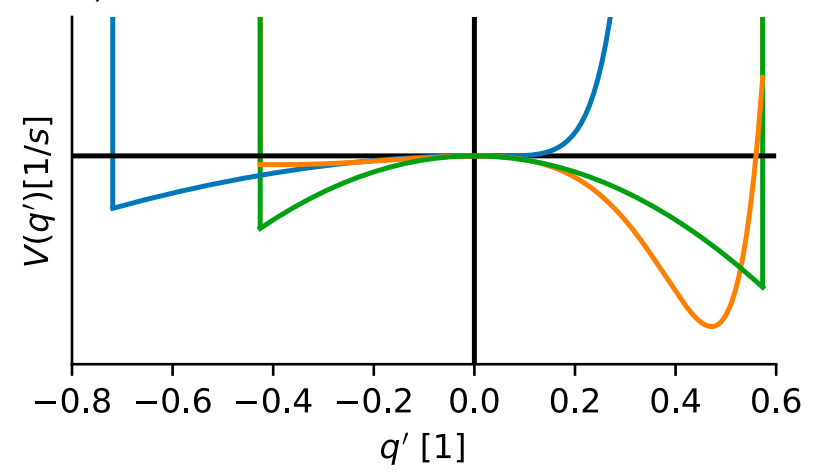

FIG. 2. (a) Tendency of humidity as a function of humidity and (b) the corresponding potential for the three models introduced by Bretherton et al. (2005) (blue), Craig and Mack (2013) (orange), and Emanuel et al. (2014) (green).

given above. ${ }^{4}$ While $R\left(q^{\prime}=0\right)=0$ shows that $q^{\prime}=0$ is a time-independent solution in each model, $\partial_{q} R\left(q^{\prime}=0\right)>0$ shows that, in all three models, any small perturbation will grow in time; that is, moist perturbations will become increasingly moist while dry perturbations become increasingly dry.

To characterize the behavior of the different feedback loops and relate them to coarsening, we compute the corresponding potential $V(q)$ for each model [see Eq. (3)]. The numerically integrated potentials are shown in Fig. 2b. The physical limits on humidity, given by complete dryness $(q=0)$ and saturation $(q=1)$, show up in the potentials as infinite walls at these humidity values. The potentials all take the double-well form that is a necessary precondition for coarsening. We can now visualize the time evolution of any humidity

\footnotetext{
${ }^{4}$ Note that while $q$ for all models represents a relative measure of humidity, that is, $q$ in $[0,1]$, the value of $q_{\mathrm{RCE}}$ depends on the model. This means that also the values of the upper and lower boundary of $q^{\prime}$, given by $q^{\prime}=1-q_{\mathrm{RCE}}$ and $q^{\prime}=-q_{\mathrm{RCE}}$, respectively, differ between the different models.
}

perturbation by "rolling down" into one of the potential minima. In particular, any initial humidity content smaller (larger) than $q_{\mathrm{RCE}}$, the local maximum of the potential, will tend to the stable dry (moist) minimum with the drying/moistening rates given by the steepness of the potential.

Comparing the three models suggests that dry perturbations always tend to an entirely dry state, while Bretherton et al. (2005) and Craig and Mack (2013) suggest that the moist equilibrium is below saturation. In fact, the moist minimum in Bretherton et al. (2005) is so close to $q^{\prime}=0$ that it is hardly visible in Fig. 2. In addition to the humidity value of the stable moist and dry state, the differences in the potentials suggest that the time evolution will be different as moistening and drying rates for a given humidity content $q$ are governed by the gradient of the potential at $q$ : the steeper the gradient, the larger the drying or moistening rate. Since the model introduced by Emanuel et al. (2014) has been linearized with respect to $q_{\mathrm{RCE}}$, the corresponding potential is symmetric with respect to $q_{\mathrm{RCE}}$ and thus moistening and drying progress at the same speed. For the model based on Craig and Mack (2013), moistening is faster than drying, in contrast to the model based on Bretherton et al. (2005), where drying is much faster than moistening. It is interesting to note that these differences between the theoretical models resemble the differences found in atmospheric models. While Wing and Emanuel (2014) and Hohenegger and Stevens (2016) find drying to be significantly faster than moistening, Holloway and Woolnough (2016) find comparable drying and moistening rates.

Representing the time evolution equations given by Eqs. (6)-(8) as double well potentials relates well to results obtained by weak temperature gradient studies that examine multiple-equilibria solutions (Sobel et al. 2007; Sessions et al. 2010, 2015, 2016; Emanuel et al. 2014). These studies suggest that, under certain conditions, the final state of a model based on the weak temperature gradient approximation depends on the initial humidity content, with dry initial conditions evolving toward a unique dry equilibrium state while moist initial conditions develop toward a unique moist equilibrium state. Comparison with the potentials introduced above suggests that the two equilibria correspond to the moist and dry potential minima.

\section{Exchange of humidity between different regions}

The positive feedback loops introduced in the previous section describe processes that locally amplify an existing humidity perturbation and do not consider any exchange of humidity between neighboring grid 
columns. Such an exchange, however, must exist to explain the formation of coherent moist and dry regions during self-aggregation. In this section, we propose that an exchange of humidity can result from convectively induced moistening.

While the relevant mechanisms in the three models introduced above differ, the cause of the local increase in humidity is convection. Bretherton et al. (2005), for example, formulate the forcing terms of their humidity equation in terms of precipitation because they argue that, on physical grounds, the forcing terms should depend on the amount of convection. The increase of humidity by convection is a central component of the model introduced by Craig and Mack (2013), and though, in the model introduced by Emanuel et al. (2014), convection is not central for the feedback loop, it is again a source of increased humidity.

This increase in humidity is not strictly local, but convection will moisten the atmosphere within a finite region around the convective updraft. Thus, even though convective updrafts preferentially occur in moist regions, convection can increase the humidity content of neighboring columns independently of whether they are dry or moist. To investigate the consequences of this, we first introduce a simple model that represents the increase in humidity within a finite region around convective updrafts as well as the increased likelihood of deep convection to occur in moist regions. We show that the result of this model is a smoothing of sharp gradients between moist and dry regions. In the second part of this section, we show that the spatial coupling resulting from the simple model of convective moistening can be represented as a reaction-diffusion equation, introduced in Eq. (4), with a strictly local increase in humidity combined with a diffusion term.

\section{a. Simple model of convective moistening}

In the following, we introduce a simple model to describe the effect of convective moistening in a spatially extended system. Our assumptions are that

1) deep convection moistens the atmosphere within a finite distance around the convective updraft (e.g., Parsons et al. 2000; Redelsperger et al. 2002) and

2) deep convection is more likely in an already moist environment (e.g., Derbyshire et al. 2004).

As we neglect any other processes that lead to an increase or loss in humidity, the humidity content at a given location depends only on the location of cloud formation and the increase in humidity around the cloud centers.

We describe the increase in humidity due to a cloud centered at $x_{c}$ in terms of the total increase in humidity

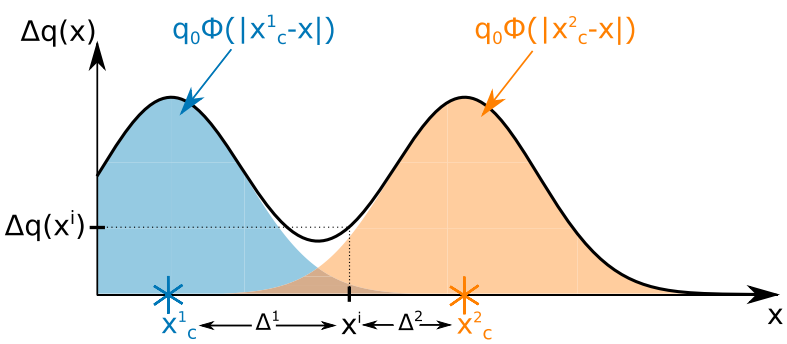

FIG. 3. Schematic showing the increase in humidity $\Delta q$ in the simple model due to two convective updrafts with centers at $x_{c}^{1}$ and $x_{c}^{2}$. As indicated by the blue and the orange areas, the increase in humidity at $x$ due to the two clouds is given by $q_{0} \Phi\left(\left|x_{c}-x\right|\right)$ and thus the total increase is given by $q_{0} \Phi\left(\left|x_{c}^{1}-x\right|\right)+q_{0} \Phi\left(\left|x_{c}^{2}-x\right|\right)$, (solid black line). The increase in humidity at $x^{i}, \Delta q\left(x^{i}\right)$, thus depends on the number of cloud centers within the surrounding of $x^{i}$ as well as their respective distance to $x^{i}$, indicated by $\Delta^{1}$ and $\Delta^{2}$.

$q_{0}$ and its spatial distribution according to a normalized function $\phi$ to which we will in the following refer as humidity distribution. In particular, the increase in humidity at a given distance from the cloud center $\left|x_{c}-x\right|=\Delta$ is described by $\phi(\Delta)$ multiplied by $q_{0}$. As multiple clouds can form during one time step, the total increase in humidity at a given location $x$ can result from multiple clouds at, or in the vicinity of, $x$. This is illustrated for two clouds in Fig. 3, where the humidity increase due to cloud 1 and cloud 2 is given by $\phi\left(\left|x_{c}^{1}-x\right|\right)$ and $\phi\left(\left|x_{c}^{2}-x\right|\right)$, respectively.

As we expect more clouds in an already moist region, the locations at which the convective updrafts form in the model should depend on humidity in such a way that the expected number of clouds within one time step and column, given by $\langle N(q)\rangle$, increases with humidity. Naturally, $\langle N\rangle$ also increases with the spatial extent of the column $d x$ and the length of the considered timestep $d t$. To separate the dependence of convective updrafts on $q$ from the dependence on $d t$ and $d x$, we introduce an average rate of occurrence of convective updrafts $n=\langle N\rangle d x^{-1} d t^{-1}$.

Based on $n[q(x)]$, which depends on $x$ through the humidity content $q(x)$, and the spatial distribution of convective moistening $q_{0} \phi$, the expected increase in humidity at a given location $x$ can be expressed by the following integral:

$$
\partial_{t} q(x)=\int_{-\infty}^{\infty} n[q(x+\Delta)] q_{0} \phi(\Delta) d \Delta
$$

where the increase in humidity at a given location $x$ not only depends on the clouds forming at $x$, that is, $n[q(x)]$, but also on the number and location of clouds that form in the surrounding of $x$, that is, $n[q(x+\Delta)]$. This is also shown schematically in Fig. 3. 
While Eq. (9) describes the expected rate of increase in humidity, we will in the following simulate the stochastic process directly. To this end, we first introduce an explicit dependence of $n(q)$ and $\phi(\Delta)$. For simplicity, we consider a linear increase of cloud number with humidity, that is, $n(q)=n_{0}+n_{1}\left(q-q_{\mathrm{RCE}}\right)$ and note that this also represents a first-order Taylor expansion of a more complicated $n(q)$ around $q_{\mathrm{RCE}}$. For the spatial distribution function $\phi(\Delta)$, we assume that the strongest increase in humidity is close to the updraft center, which we represent using a normalized Gaussian

$$
\phi(\Delta)=\frac{1}{2 \pi r_{\mathrm{fp}}^{2}} e^{-r^{2} /\left(2 r_{\mathrm{fp}}^{2}\right)}
$$

where the radius $r_{\mathrm{fp}}$ determines the width of the humidity distribution.

Before we can simulate the above described model, we need to estimate the following parameters:

- $r_{\mathrm{fp}}$ : Width of the humidity distribution

- $q_{0}$ : The total increase in humidity associated with one cloud

- $q_{\mathrm{RCE}}$ : The mean humidity content in RCE in the absence of self-aggregation

- $n_{0}$ : The average number of clouds per time and area at $q_{\mathrm{RCE}}$

- $n_{1}$ : The additional number of clouds per time, area, and $\Delta q$ at $q_{\mathrm{RCE}}+\Delta q$

To estimate the radius $r_{\mathrm{fp}}$, we assume that the width of the humidity distribution is related to the typical extent of deep convective updrafts. In particular, we choose $r_{\mathrm{fp}}$ such that the region $r \leq r_{\mathrm{fp}}$, where humidity increases strongly, corresponds to the typical extent of a deep convective updraft in the free troposphere. As Doswell (1985), studying the life cycle of a common convective shower, find updrafts on the order of $5 \mathrm{~km}$ during the early and mature stage of the life cycle, we set the radius $r_{\mathrm{fp}}=2.5 \mathrm{~km}$. Note that in setting $r_{\mathrm{fp}}$ equal to $2.5 \mathrm{~km}$, $99.5 \%$ of the increase in humidity given by Eq. (10) happens within a region with a horizontal extent of $15 \mathrm{~km}$, a typical spatial extent of a deep convective cloud in its dissipating stage (Doswell 1985). For the total increase in humidity associated with one cloud $q_{0}$, we assume that the cloud initially increases the humidity from $q_{\mathrm{RCE}}$ to saturation (i.e., $q=1$ ) within $r_{\mathrm{fp}}$ throughout the free troposphere, which gives $q_{0}=\left(1-q_{\mathrm{RCE}}\right) \pi r_{\mathrm{fp}}^{2}=5.5 \times 10^{6} \mathrm{~m}^{2}$. To estimate the average number of clouds $n_{0}$ per area and time, we assume that at any given time the cloud fraction $c_{f}$ is about $10 \%$ (e.g., Stein et al. 2017), which, together with $r_{\mathrm{fp}}=2.5 \mathrm{~km}$ and an average cloud lifetime $c_{t}$ of $1 \mathrm{~h}$, gives $n_{0}=c_{f} /\left(\pi r_{f p}^{2} c_{t}\right)=1.4 \times 10^{-12} \mathrm{~m}^{-2} \mathrm{~s}^{-1}$. Finally, to

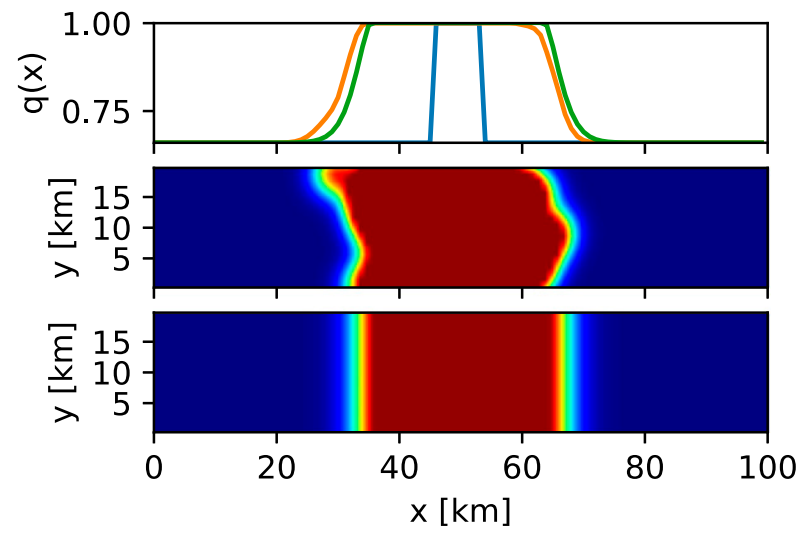

FIG. 4. (top) Initial humidity profile (blue) and humidity profiles after $10 \mathrm{~h}$ for the simple convective moistening model (orange) and the corresponding deterministic model (green). (middle),(bottom) Snapshots of the simple convective moistening model and the deterministic model after $10 \mathrm{~h}$, respectively.

estimate the increase in cloud number with humidity $n_{1}$, we use the dependency of precipitation on humidity given in Eq. (5) and Table 1. Assuming that precipitation is a proxy for the amount of convection, we estimate $n(q)=n_{0} \exp \left[k_{1}^{b}\left(q-q_{\mathrm{RCE}}\right)\right] \approx n_{0}+n_{0} k_{1}^{b}\left(q-q_{\mathrm{RCE}}\right)$; that is, $n_{1}=n_{0} k_{1}^{b}=2.3 \times 10^{-11} \mathrm{~s}^{-1} \mathrm{~m}^{-2}$. Note that this approximation should be considered as an order of magnitude estimate because the expansion of the exponential pickup curve gives good agreement only close to $q_{\mathrm{RCE}}$.

Starting from a humidity field with a step function profile in the $x$ direction, as can be seen in the top panel of Fig. 4 , a cloud is assigned with probability $n[q(i, j)]$ at every site $(i, j)$. Here, we choose that, during each time step, a maximum of one cloud can form per grid cell as the chosen horizontal resolution of $1 \mathrm{~km}$ and the corresponding time step of $60 \mathrm{~min}$ allow for only one deep convective cloud at a time. Note that we enforce a lower and an upper limit on the humidity content of $q_{\mathrm{RCE}}-1 / k_{1}^{b} \leq q \leq 1$. While the upper limit is a physical limit, the lower limit ensures that the rate of clouds cannot become negative. If a cloud is assigned to site $(i, j)$, its humidity together with the humidity of the surrounding cells is increased by $q_{0} \phi\left(\left|r-r_{(i, j)}\right|\right)$. The profile of a sample model output after $10 \mathrm{~h}$ is shown in addition to the initial profile in the top panel of Fig. 4 and the corresponding snapshot in the middle panel. The front between the moist and the dry regions after $10 \mathrm{~h}$ has propagated into the dry regions and the gradient has been smoothed.

\section{b. Deterministic approximation}

In the following, we will show that the model described by Eq. (9) can be approximated by a reactiondiffusion equation. Using, as above, a first-order Taylor expansion of $n(q)$ and in addition a second-order Taylor expansion of $q(x+\Delta)$, Eq. (9) can be written as 


$$
\begin{aligned}
\partial_{t} q(x) & \approx\left[n_{0}+n_{1} q(x)\right] q_{0} \underbrace{\int_{-\infty}^{\infty} \phi(\Delta) d \Delta}_{=1}+n_{1} q_{0} \partial_{x} q \underbrace{\int_{-\infty}^{\infty} \Delta \phi(\Delta) d \Delta}_{=0}+\frac{1}{2} n_{1} q_{0} \partial_{x}^{2} q \underbrace{\int_{-\infty}^{\infty} \Delta^{2} \phi(\Delta) d \Delta}_{\text {Depends only on } \phi} . \\
& =\left[n_{0}+n_{1} q(x)\right] q_{0}+D \partial_{x}^{2} q \quad \text { with } \quad D \equiv \frac{1}{2} n_{1} q_{0} \int_{-\infty}^{\infty} \Delta^{2} \phi(\Delta) d \Delta,
\end{aligned}
$$

where the integral in the first term evaluates to one because of the normalization constraint on $\phi(\Delta)$, the integral in the second term vanishes as we integrate an antisymmetric function over a symmetric interval, while the integral in the last term is determined by the choice of $\phi(\Delta)$.

Equation (12) has the same functional dependence as a reaction-diffusion equation. The source term $R$ is given by $\left[n_{0}+n_{1} q(x)\right] q_{0}$ and the diffusive part by $D \partial_{x}^{2} q(x, t)$. This derivation is similar to Einstein's famous "proof" of the existence of Brownian motion (Einstein 1905). It runs analogously in the two-dimensional case.

To obtain a simple estimate for $D$, we assume $\phi(\Delta)$ is given by Eq. (10). The 2D diffusion coefficient is then given by

$$
D=\frac{n_{1} q_{0}}{2} r_{\mathrm{fp}}^{2}
$$

Using Eq. (13) and the parameters estimated above results in $D=4.0 \times 10^{2} \mathrm{~m}^{2} \mathrm{~s}^{-1}$.

Starting from the same initial humidity profile previously used for the simple model of convective moistening, a snapshot of the time evolution after $10 \mathrm{~h}$ of this deterministic model is shown in the bottom row of Fig. 4 with the corresponding humidity profile shown in the top row. It resembles the evolution of the previously introduced model but is completely symmetric in the $y$ direction, without any fluctuations in propagation speed and boundary width as found in the simple convective moistening model.

To summarize, Eq. (12) shows that the coupling of the humidity content induced by convective moistening can be approximated by a strictly local increase of humidity due to the number of clouds at that location and a diffusion term that spreads humidity in space. While the model of convective moistening presented here thus motivates the use of a diffusion term in the following, we note that the derivation above should not be considered as a proof for a diffusive exchange of humidity in RCE simulations as our model neglects any other processes that can contribution to the horizontal exchange of humidity. One potentially relevant process is the upgradient transport of humidity due to a radiatively driven shallow circulation (e.g., Muller and Held 2012). As we will discuss in section 5 below, this process becomes increasingly important with time and thus suggests that diffusion might be a good approximation of the horizontal exchange mainly during the early stages of selfaggregation.

\section{Spatial evolution}

Section 3 shows that the moistening of the atmosphere within finite regions around convective updrafts can be approximated by a reaction-diffusion equation. The model described by Eq. (12) so far only includes the tendency of the atmosphere to moisten regions of increased convection, but we know from section 2 that there exists a positive feedback where not only moist regions get moister but also dry regions drier. As we have seen that the three local source terms $R_{B}$ [Eq. (6)], $R_{C}$ [Eq. (7)], and $R_{E}$ [Eq. (8)] can be described as derivatives of a double-well potential, combining them with a diffusion term leads to three TDGL equations [Eq. (3)].

Starting from a homogeneous state of $q=q_{\mathrm{RCE}}$, perturbed with spatially uncorrelated noise drawn from a uniform distribution with amplitude of \pm 0.05 , we now examine the time evolution of two-dimensional humidity fields resulting from the three models. The time evolution is calculated numerically for a doubly periodic domain, using second-order finite differences in space and a fourth-order Runge-Kutta method in time. If not stated otherwise, results will be shown for a domain of size of $600 \mathrm{~km} \times 600 \mathrm{~km}$ with a horizontal resolution of $5 \mathrm{~km}$.

\section{a. Snapshots}

Snapshots from all three models are shown in Fig. 5, where we distinguish only between locations that are drier or moister than the initial state $q_{\mathrm{RCE}}$ :

$$
q(x, y)=\left\{\begin{array}{ll}
0 & \text { if } q \leq q_{\mathrm{RCE}} \\
1 & \text { if } q>q_{\mathrm{RCE}}
\end{array} .\right.
$$

We focus on these two states as coarsening describes the spatial evolution of the moist/convecting and the dry/nonconvecting phases rather than details of moisture variability. While the moisture variability in the theoretical 

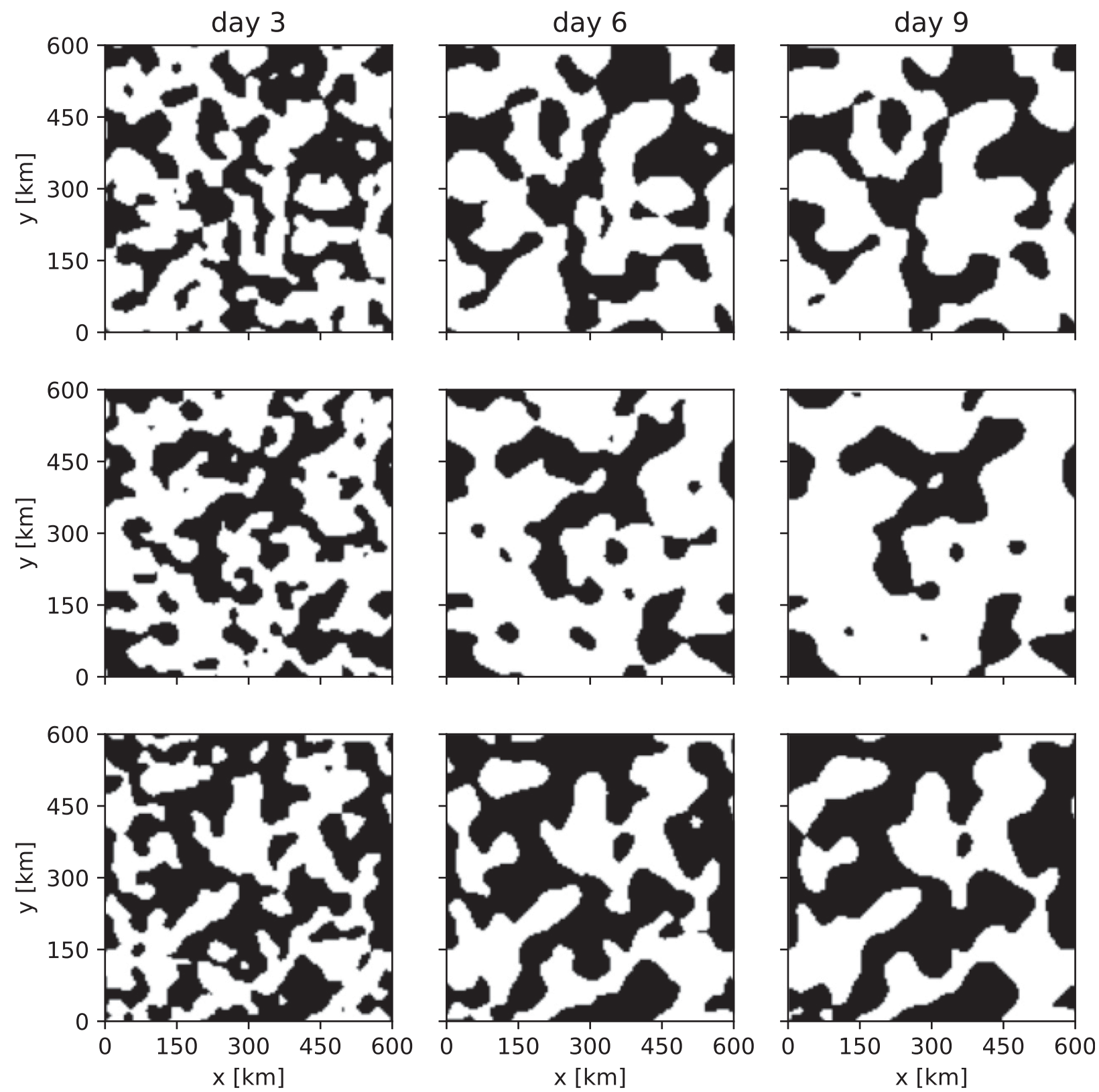

FIG. 5. Time evolution of regions of dry (white) and moist (black) perturbations. Results for a sample output from the model based on (top) Bretherton et al. (2005), (middle) Craig and Mack (2013), and (bottom) Emanuel et al. (2014).

models results from the moistening and drying rates given by the respective potentials, moisture variability in atmospheric models can also be produced by processes not represented in the theoretical models, for example, propagating gravity waves or individual deep convective updrafts.

In Fig. 5, the moist and dry regions of each model show an upscale growth of the typical length scale with time, as characteristic for coarsening. In particular, the upscale growth can be described as a successive disappearance of small-scale features and thus a smoothing of the boundaries between the two regions. While the snapshots in Fig. 5 show a roughly equal partitioning into moist and dry regions, we note that for all models one phase eventually becomes dominant and covers the entire domain. In fact, the dry regions already start to dominate over the moist regions in the snapshot corresponding to the model based on Craig and Mack (2013), see middle row of Fig. 5. A uniform moist or dry state is possible here because, in contrast to Craig and 
a)

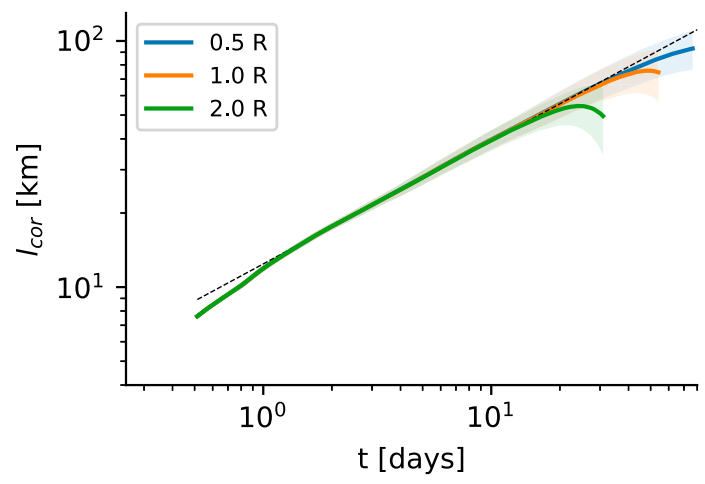

c)

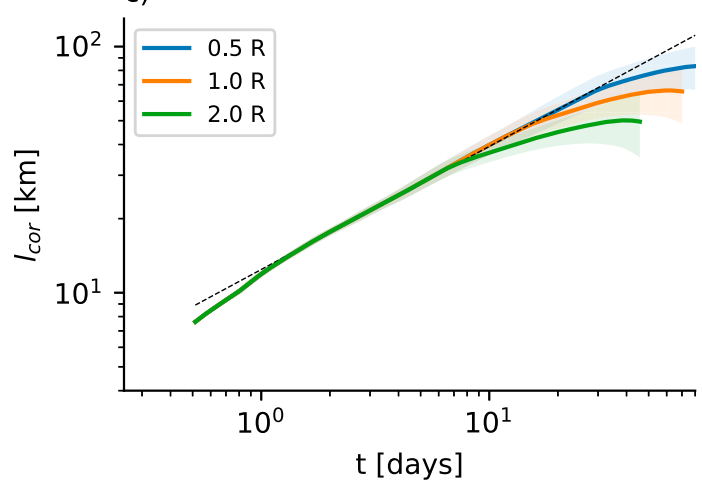

b)

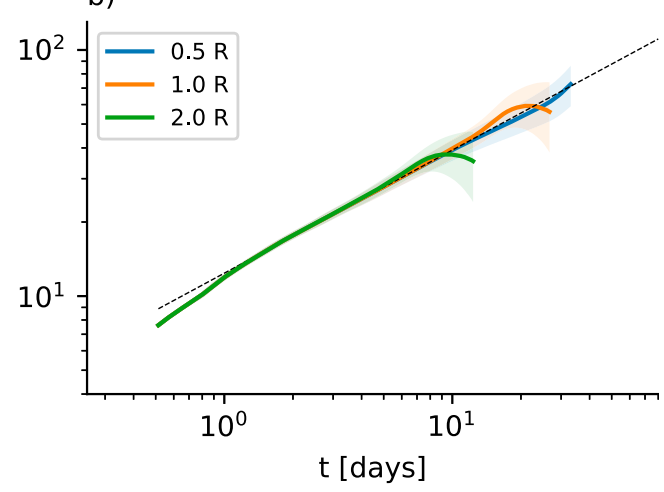

d)

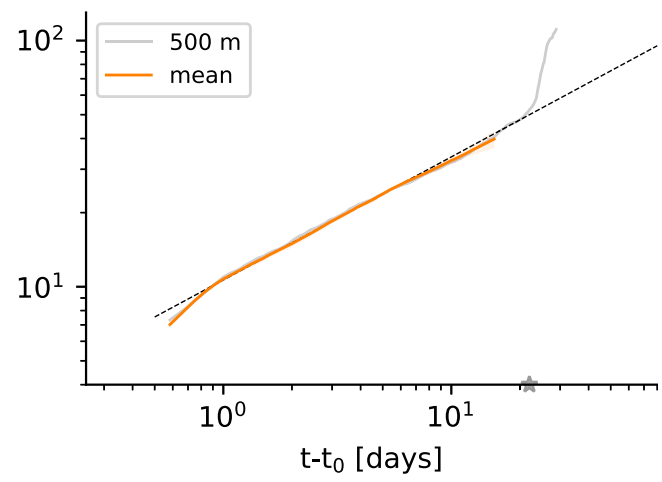

FIG. 6. Scaling of the average autocorrelation length with time for the three different models: (a) Bretherton et al. (2005), (b) Craig and Mack (2013), (c) Emanuel et al. (2014), and (d) for a set of RCE simulations with varying slab-ocean depth performed by Hohenegger and Stevens (2016), see text for details. In (a)-(c) the different colors mark different feedback strengths and the shaded area indicates the standard deviation. In (d), the autocorrelation length of just the 500-m slab-ocean run is included in addition to the average scaling, and the time axis has been shifted by $t_{0}=1$ day to exclude the time before self-aggregation has started (see text for further details). Each panel also shows the theoretically expected dependence (dashed line) with the star in (d) marking the position where the $500-\mathrm{m}$ slab-ocean run starts to strongly deviate from this scaling.

Mack (2013), we have not included a global constraint on the amount of convection.

Note that even though the spatial evolution of regions either moister or drier than the equilibrium humidity content in Fig. 5 is very similar for the three models, the actual humidity content within these regions (not shown) reflects the differences in the potentials.

\section{b. Scaling}

One of the central properties of coarsening is dynamical self-similarity, where the increase in typical feature size is proportional to $t^{1 / 2}$. To compare the upscale growth in feature size shown in Fig. 5 with the expected scaling, Figs. $6 \mathrm{a}-\mathrm{c}$ show the average scaling of the autocorrelation length $l_{\text {cor }}$, a measure for the typical feature size, with time for all three models. The autocorrelation length is calculated on the binary fields [see Eq. (14)] with details on the calculation given in the supplementary material. For each model, the average and standard deviation of 512 runs is calculated. The only difference between the model runs is different random noise in the initial field. Each panel of Fig. 6 has a double-logarithmic scale, so that a power-law dependence will appear as a straight line. Dotted lines show the expected $t^{1 / 2}$ scaling for coarsening. We find that, after an initial transition phase, the time evolution of the autocorrelation length in all three models is well described by the expected scaling, during the formation and the upscale growth of moist and dry regions. But for all models, the scaling eventually diverges from the $t^{1 / 2}$ scaling. This departure from the expected scaling appears to start when either the moist or dry regions begin to cover significantly more than half of the domain, that is, when the moist (dry) regions start to expand at the expense of the dry (moist) regions.

The scaling of the upscale growth of moist and dry regions is the most important prediction from coarsening. In the following, we will show how the scaling in 
Figs. $6 a-c$ depends on the strength of the feedback and the diffusion coefficient and compare the different models to note the impact of different potential shapes.

\section{1) SEnsitivity TO FEEDBACK STRENGTH}

In addition to the model parameters estimated in section 2, Figs. 6a-c show the scaling resulting from models with changed feedback strength. In particular, we multiply the reaction term $R$ for each model [Eqs. (6)-(8)] by a constant value to make the moistening/drying tendencies half (indicated in the legend as $0.5 R$ ) or twice as fast $(2.0 R)$. Figure 6 shows that the main impact on the scaling is that weaker (stronger) feedbacks lead to longer (shorter) $t^{1 / 2}$ scaling, while there is little impact on the initial evolution and the spatial extent of the features at a given time.

\section{2) SENSITIVITY TO DIFFUSION COEFFICIENT}

A change in the diffusion coefficient changes the typical length scale by a constant factor. This can immediately be shown as the $2 \mathrm{D}$ version of Eq. (3) can, without loss of generality, be rewritten as $\partial_{t} q(\tilde{x}, \tilde{y})=R[q(\tilde{x}, \tilde{y})]+\left(\partial_{\tilde{x}}^{2}+\partial_{\tilde{y}}^{2}\right) q(\tilde{x}, \tilde{y})$ with $\tilde{x}=\sqrt{D} x$ and $\tilde{y}=\sqrt{D} y$. This shows that an increase in the diffusion constant $D$ leads to an increase in all spatial scales by a constant factor, including the autocorrelation length. As the dependence of the autocorrelation length on time is shown in a double-logarithmic plot in Fig. 6, a change in diffusion constant thus results in a shift along the $y$ axis but leaves the functional dependence unchanged. In addition to the rescaling of the general length scale, we note that the initial rapid growth is determined by the diffusion term acting on the randomly perturbed initial field. As we have used the same diffusion coefficient for the three models, the evolution up to the departure from the scaling is essentially identical for all models. This can be seen in Figs. 6a-c, where the autocorrelation length is similar for the different models, and also when comparing the size of the spatial structures in Fig. 5.

Changing the feedback strength and/or the diffusion coefficient affects the nonuniversal aspects of the scaling: the initial transition phase, the typical feature size, and the time at which the scaling starts to deviate from the expected scaling. In contrast, the universal property of $t^{1 / 2}$ scaling is not affected by a change in feedback strength or diffusion coefficient. The distinction between universal and nonuniversal properties will be important for the following discussion, where we compare the theoretical models with atmospheric model simulations. On the one hand, we expect that if selfaggregation is described by coarsening, the upscale growth of moist and dry regions should follow the universal $t^{1 / 2}$ scaling even if none of the theoretical models exactly captures the feedback acting in the atmospheric model. On the other hand, the nonuniversal properties of coarsening depend on the mechanisms driving self-aggregation. As stated in the introduction, the mechanisms driving selfaggregation differ between atmospheric simulations, and we therefore expect that the nonuniversal properties of coarsening will depend on the details of how the simulations are configured.

\section{Comparison of coarsening and self-aggregation in RCE simulations}

The results from the previous section suggests that the spatial evolution of self-aggregation of convection in RCE simulations might be explained by coarsening, independent of the processes that lead to the positive feedback. In this section, we will compare properties of coarsening with properties observed in RCE simulations.

\section{a. Upscale growth}

Figure 7 shows snapshots of the dry and moist regions in the RCE simulation described in Hohenegger and Stevens (2016). The atmospheric model simulation was performed with the University of California, Los Angeles, Large-Eddy Simulation model coupled to a slab ocean with a depth of $500 \mathrm{~m}$, a horizontal domain size of $576 \mathrm{~km} \times 576 \mathrm{~km}$, and a spatial resolution of $3 \mathrm{~km}$; see Hohenegger and Stevens (2016) for more details on the simulation. Dry (moist) regions in Fig. 7 corresponds to regions where the water vapor path (WVP) is smaller (larger) than the equilibrium WVP $(41.6 \mathrm{~mm})$, which we define as the mean humidity content of the domain before the effect of domainwide drying (due to self-aggregation) sets in. As expected for coarsening and as seen for the theoretical models in Fig. 5, coherent moist and dry regions form and grow upscale in time.

It is interesting to note that the upscale growth of moist and dry regions in Fig. 7 happens, as in the theoretical models, simultaneously with a comparable area covered by moist and dry regions. As the final state of self-aggregation, however, is marked by a mainly dry region, we note that the dry regions have eventually to start growing at the expense of the moist regions. Such an expansion of dry regions at the expense of moist regions has been previously explained by Coppin and Bony (2015) through a process other than coarsening. Investigating self-aggregation in a general circulation model, Coppin and Bony (2015) showed that, at low SSTs, strong radiative cooling at the low-level cloud tops in dry regions leads to the formation of density currents. These density currents increase the size of the dry regions by exporting humidity out of these regions. As 

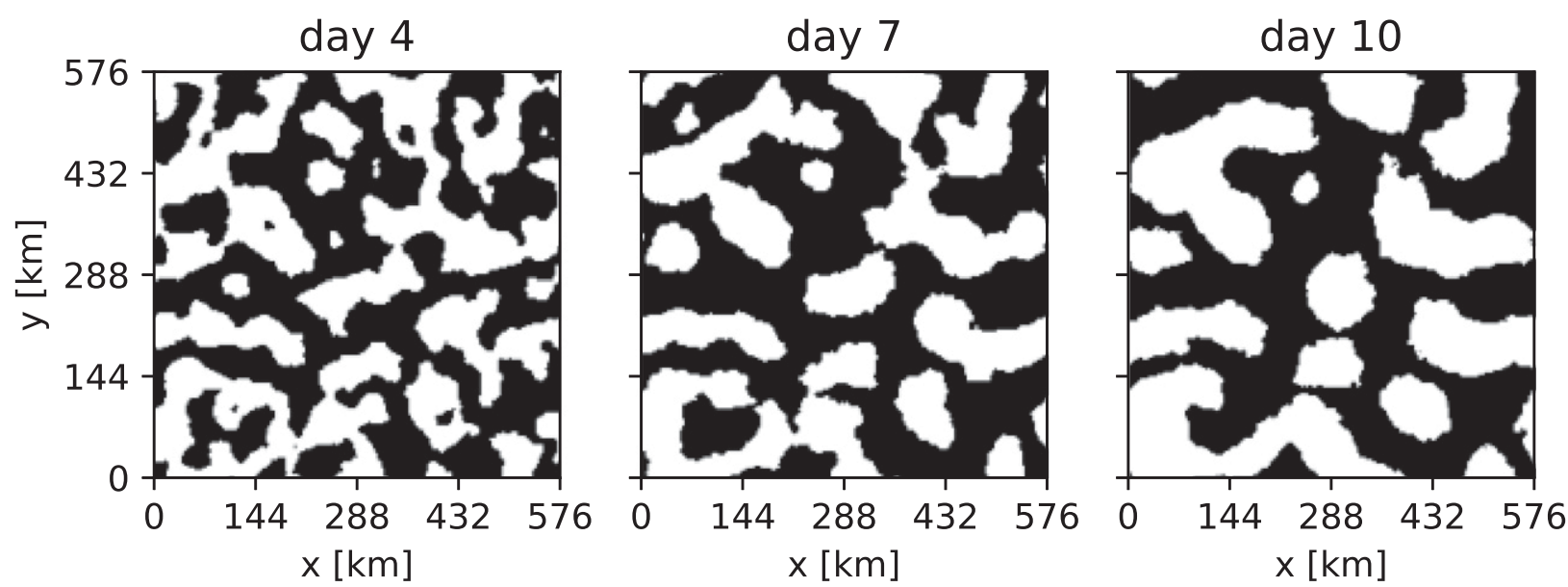

FIG. 7. Snapshots of the moist (dry) regions in an RCE simulation performed by Hohenegger and Stevens (2016), with moist (dry) defined by WVP $>41.6 \mathrm{~mm}(\mathrm{WVP} \leq 41.6 \mathrm{~mm})$. See text for details.

noted in the introduction, advection of humidity out of dry regions differs from the diffusive transport of humidity in the models proposed here. In the next section, we will present some indications for a transition from a diffusion- to an advection-dominated stage of selfaggregation.

An equal partitioning into moist and dry regions during the onset of self-aggregation as in Fig. 7 is not as clear in other RCE studies (e.g., Bretherton et al. 2005; Wing and Cronin 2016). A possible explanation for this could be a strong asymmetry between the moistening and drying rates. On the one hand, this could make the upscale growth of one phase more apparent than the other phase; in this case, viewing the regions that contain deep convection should give a more equally distributed appearance [which seems to be the case in, e.g., Bretherton et al. (2005)]. On the other hand, the asymmetry could be so strong as to lead to a discernible imbalance between the two regions early in the self-aggregation process (e.g., Wing and Cronin 2016).

\section{b. Scaling in RCE simulations}

We now determine whether the observed upscale growth in moist and dry regions agrees with the $t^{1 / 2}$ scaling expected for coarsening. Figure $6 \mathrm{~d}$ shows the autocorrelation length as a function of time in a doublelogarithmic plot for the simulation shown in Fig. 7. In addition, we also include the average autocorrelation length and the corresponding standard deviation, calculated from a set of seven simulations performed by Hohenegger and Stevens (2016). The simulations differ in the slab-ocean depth and therefore in the ocean heat capacity, instead of only differing in the initial conditions as in the theoretical models. As we however want the simulations to be as comparable as possible to each other, we do not average over all simulations presented in Hohenegger and Stevens (2016) but restrict our analysis to slab-ocean depths of at least $25 \mathrm{~m}$ as the onset of SA gets delayed for shallower ocean depths [see Hohenegger and Stevens (2016) for more details]. Note that self-aggregation does not start immediately but needs some time to trigger. This results in a time period during which we do not expect coarsening to apply. We therefore shift the time of the simulation with respect to this offset time. The offset time $t_{0}$ for the shown snapshots and scaling was determined from the frequency plot in Hohenegger and Stevens (2016), which suggests that self-aggregation is present after the first day (see their Fig. 1), that is, $t_{0}=1$ day. Note that we do not attempt to explain this delay in the start of self-aggregation in this study but discuss a possible explanation in the conclusions.

For the first day, the single and the average autocorrelation length grow more rapidly than is compatible with the $t^{1 / 2}$ scaling we expect for coarsening. It is interesting to note that a rapid initial growth is also apparent for the simple models. After this initial phase, the average autocorrelation length follows the expected scaling until the end of the simulation period of the set of the simulations ( 15 days) though with a slowly increasing deviation apparent after approximately day 8 . This is also seen in the $500-\mathrm{m}$ simulation (which is integrated longer than the others), but there is also a dramatic deviation from the expected scaling after about 23 days (marked by a star in Fig. 6d). These results suggest that coarsening is important during the early stages of self-aggregation but may become less important at later times.

In the following, we present some indications that this departure in the scaling results from a transition from 
self-aggregation driven by local mechanisms to selfaggregation driven by advective mechanisms. As discussed in the introduction, coarsening requires local mechanisms, that is, mechanisms leading to an amplification of local humidity perturbations, and a diffusive and thus downgradient transport of humidity. While studies quantifying the importance of different processes [using the diagnostic introduced by Wing and Emanuel (2014) and described in the introduction] have shown that local mechanisms are most important during the early stages of self-aggregation, advective mechanisms marked by an upgradient transport of humidity become important during the intermediate stage of self-aggregation (Wing and Emanuel 2014; Holloway and Woolnough 2016). As the upgradient transport of humidity is caused by a shallow circulation, with low-level inflow from the dry to the moist regions, we use the low-level velocity as an indication for the importance of advection. Figure 8 therefore shows the near-surface velocity averaged over a narrow stripe along the border of the moist and the dry regions as a function of time. We find that the average wind speed systematically increases with time, where a slow increase until about day 20 is followed by a more rapid increase thereafter. In agreement with our hypothesis, we find that a rapid pickup of the horizontal wind speed appears at a similar time as the above-noted deviations from the expected scaling for the correlation length (day 23).

The evolution of self-aggregation after day 23 is no longer described by coarsening but might be described by radiatively driven pools, introduced by Coppin and Bony (2015) and reviewed above. An increase in lowlevel wind speed at the border of the dry region with time is indeed apparent in Fig. 4 of Coppin and Bony (2015), suggesting that the expansion of dry regions through radiatively driven cold pools becomes stronger with time. A transition to an advection-dominated stage of self-aggregation cannot be represented by the theoretical models introduced here, which suggests that the upscale growth in the RCE studies and the theoretical models deviate from the expected scaling for different reasons. This might explain why the upscale growth in Fig. 6 changes in different ways; while it slows down in the theoretical models (Figs. 6a-c), it accelerates in case of the RCE study (Fig. 6d).

In another RCE simulation, performed by Wing and Cronin (2016), they find scaling of the autocorrelation length with time but with an exponent of approximately one. We note, however, that they used a very elongated, channel-like domain, which led to the formation of persistent moist and dry stripes, and thus a quasi-onedimensional humidity field, while power-law scaling with an exponent of $1 / 2$ is only expected for dimensions

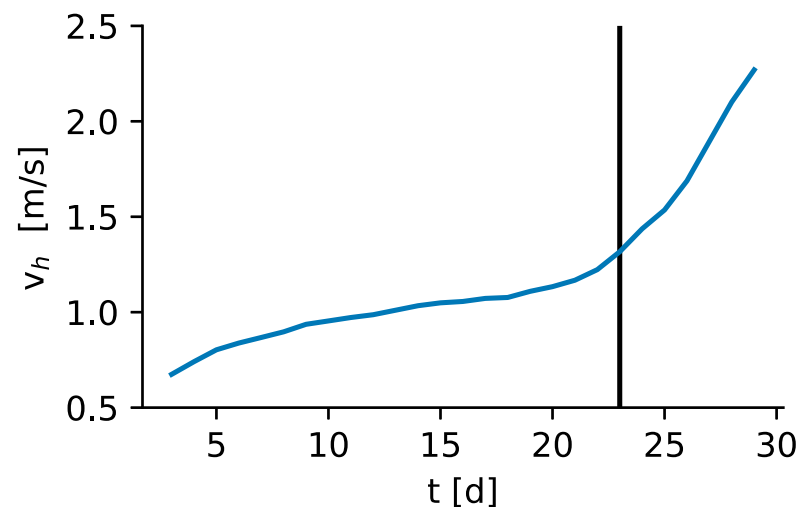

FIG. 8. Average horizontal wind speed at the boundary of the moist and dry regions at a height of $150 \mathrm{~m}$ for the $500-\mathrm{m}$ slab-ocean run described in Hohenegger and Stevens (2016). The vertical line denotes the time where the corresponding scaling, shown in Fig. 6, starts to strongly deviate from the scaling expected from coarsening.

$d \geq 2$. This distinction between one dimension and all higher dimensions arises because of the absence of curvature in one dimension, which can be briefly explained as follows. The time evolution of the interface between the moist and dry regions for dimensions $d \geq 2$ can be thought of as driven by surface tension (see, e.g., Sethna 2006). As surface tension increases with curvature, the smallest features disappear quickest for $d \geq 2$. The absence of curvature in the one-dimensional case makes the resulting interface velocity, and therefore the upscale growth of features, very slow [it can in fact be shown analytically that, in one dimension, the scaling is given by $L(t) \sim \ln (t)]$. After the formation of moist and dry bands, the contribution to the spatial evolution from coarsening is therefore probably small and the scaling exponent found by Wing and Cronin (2016) a consequence of an advection-dominated regime.

\section{c. Domain-shape dependence}

The appearance of SA is strongly influenced by the shape of the domain, with the final shape of the moist region found to be either band-like or circular. Which of these two is realized seems to depend on whether the domain is channel-like or square. While channel-like domains always result in moist bands (e.g., Tompkins 2001; Posselt et al. 2012; Wing and Cronin 2016), square domains generally lead to the formation of circular moist regions (e.g., Bretherton et al. 2005; Muller and Held 2012; Kempf 2013; Jeevanjee and Romps 2013; Holloway and Woolnough 2016; Hohenegger and Stevens 2016), though band-like structures have (at least transiently) also been found in some studies with square domains (Tompkins and Craig 1998; Wing and Emanuel 2014; Muller and Bony 2015). 
Holloway and Woolnough (2016) first pointed out that this dependence on the shape of the domain is explained if SA tends to minimize the length of the boundary. Assuming a moist region of area $A^{\text {moist }}$, they showed that for a domain with the shortest domain side of length $L$, there exists a critical domain length $L_{c}=\sqrt{\pi A^{\text {moist }}}$ below which the minimal boundary length is given by a moist band rather than a moist circle.

A minimization of the boundary length is exactly what we expect from coarsening, where the stable solution must minimize the Landau free energy given in Eq. (2). This can be seen by noting that the free energy is larger for values of $q$ that lie between the two minima than it is for $q$ at the minima themselves. Reducing the length of the boundary corresponds to replacing intermediate values of $q$ with dry or moist values where the potential is at a minimum, thus reducing the area integral of the potential and the free energy.

As explained above, our models are intended to represent a small subdomain of a larger domain, and we therefore have no constraint on the total amount of convection (as is the case in RCE), so our domains are allowed to become completely moist or completely dry. Nevertheless, the tendency of coarsening to favor banded rather than circular regions in a channel-like domain can already be seen during the evolution. Figure 9 shows a snapshot of the model based on Craig and Mack (2013) in a channel-like domain after 6 days. Apart from the domain size, the simulation is identical to the simulation shown in Fig. 5 but leads to the formation of multiple moist and dry bands, while for a square domain with constraint on the total amount of convection, Craig and Mack (2013) show that the final state corresponds to a single, circular moist region surrounded by a dry region (their Fig. 1f).

As an alternative to the minimization of the boundary length discussed here, the existence of a typical horizontal scale of self-aggregation (e.g., Yang 2018b) could also explain the occurrence of banded structures in channel-like simulations. In that case, the banded structure could result from $L$ being smaller than the typical scale of self-aggregation. These two explanations could be tested by performing a series of RCE simulations with different domain shapes. In particular, starting from a channel-like simulation with a single moist band, $L_{c}$ could be determined by systematically increasing the short dimension of the domain until the steady state corresponds to a circular rather than a banded moist region. If the transition is due to the boundary length minimization, $L_{c}$ should be comparable with $\sqrt{\pi A^{\text {moist }}}$, while, if it is due to a typical horizontal scale, the transition should occur when $L$ becomes comparable to this scale.

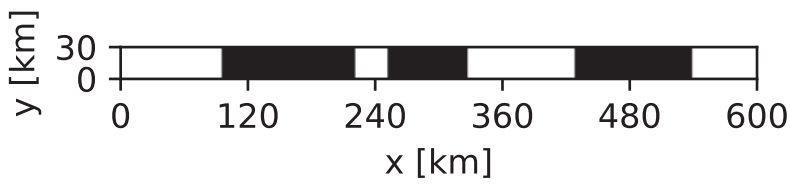

FIG. 9. Snapshot of simulation based on Craig and Mack (2013) after 6 days in channel-like domain.

As noted above, some RCE studies found (at least transiently) the formation of banded rather than circular moist regions (Tompkins and Craig 1998; Wing and Emanuel 2014; Muller and Bony 2015). While in Tompkins and Craig (1998), this is likely caused by the comparatively small domain size, the formation of banded structures in Wing and Emanuel (2014) and Muller and Bony (2015) indicates again that the final stage of self-aggregation can only partly be explained by coarsening.

\section{d. Domain-size dependence in RCE simulations}

As mentioned in section 1, the barrier width separating moist and dry regions in coarsening is completely determined by the potential and the diffusion coefficient and should thus be independent of the domain size. As we show in the following, this might explain the sensitivity of self-aggregation to domain size that was found by Bretherton et al. (2005), Muller and Held (2012), and Jeevanjee and Romps (2013), who show that selfaggregation only occurs for domains greater than $200-300 \mathrm{~km}$, though in case of Jeevanjee and Romps (2013), this domain-size dependence disappears if the formation of cold pools is suppressed.

If, in a domain with periodic boundary conditions, the domain size is on the order of the barrier width or smaller, diffusion is too strong, and the two stable regions can no longer occur within the same, periodic, region. As in RCE, the domain can become neither completely dry nor completely moist, and diffusion will tend to a homogeneous humidity content within the domain. This can be interpreted as diffusive mixing acting over a scale comparable to the domain size and thus preventing upscale growth.

An example for this is shown in Fig. 10, where we initialize two simulations by the humidity distributions shown in the top row. While the final state of the models introduced here is not constrained to have a moist and a dry region, we can ensure the presence of both regions by on the one hand setting $q_{\mathrm{RCE}}$ to 0.5 in the model based on Eq. (8), which makes the potential symmetric and the minima degenerate and thus avoids the stable region propagating into the metastable region (see introduction). On the other hand, we choose a channel-like setup for which the upscale growth becomes 

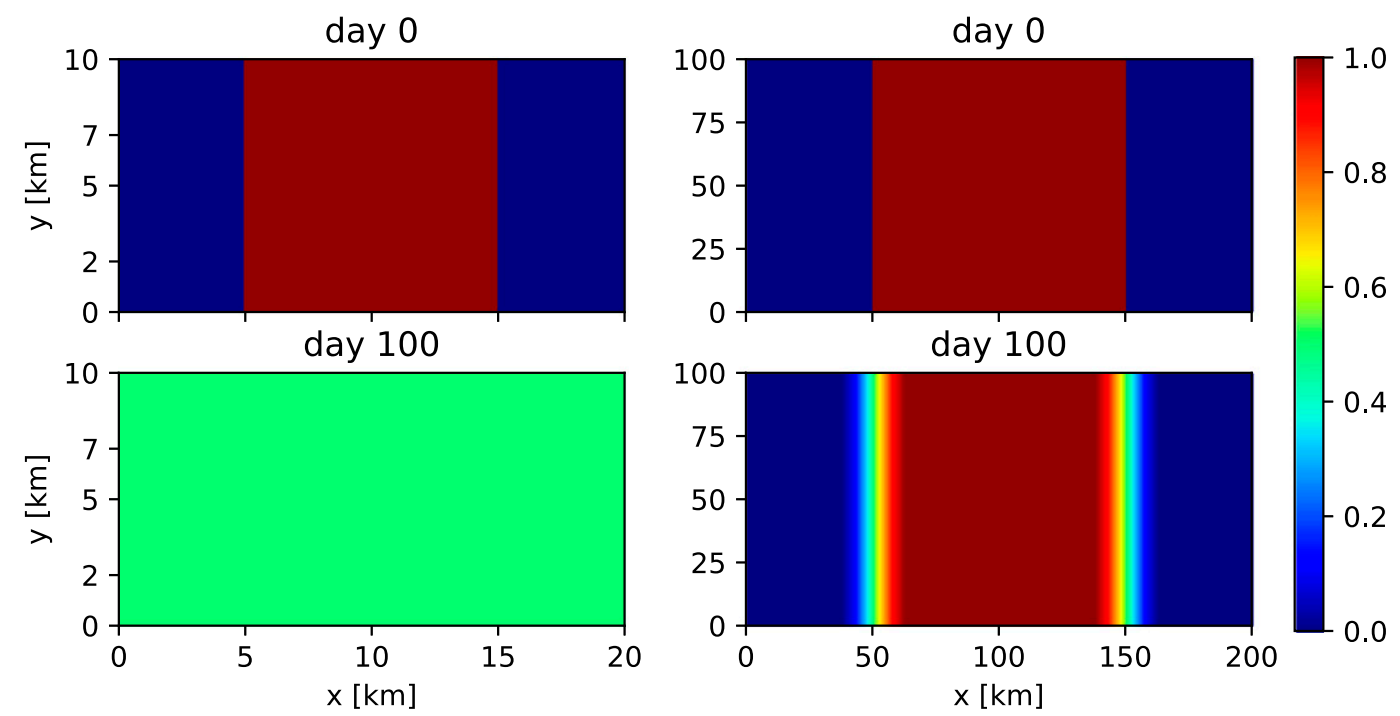

FIG. 10. (top) Initial humidity distribution for two numerical simulations, identical apart from domain size.

(bottom) Corresponding humidity fields after 100 days.

very slow once moist bands have formed (see section $5 b$ ). The only difference between the two simulations is the domain size, which is $10 \mathrm{~km} \times 20 \mathrm{~km}$ in the left panel and $100 \mathrm{~km} \times 200 \mathrm{~km}$ in the right panel. After 100 days, the larger domain is separated into a single moist and a single dry region, now separated by a smooth domain wall, while the smaller domain has evolved to a completely homogeneous state of $q=q_{\mathrm{RCE}}$. Note that increasing the diffusion coefficient, rather than reducing the domain size, eventually also leads to a homogeneous state (not shown). Considering this, the disappearance of the domain-size dependence in the absence of cold pools (Jeevanjee and Romps 2013) might suggest that cold pools increase the effective diffusion coefficient, for example, by causing the formation of convection farther away from favorable regions. Cold pools have, however, been shown to impact not only the domain-size dependence of self-aggregation but also, for example, its dependence on radiation (Muller and Bony 2015; Yang 2018a), which suggests that cold pools or, more precisely, the evaporation of raindrops in the boundary layer impact more than just the diffusivity.

Though beyond the scope of this study, we note that the above-given explanation could be tested by conducting a series of experiments with increasing domain size. In particular, one could use these experiments to first determine whether the barrier width is really independent of the domain size and second to determine whether self-aggregation disappears if the domain size becomes comparable to 2 times the barrier width.

In addition to the domain-size dependence discussed here, Muller and Held (2012) found also a dependence on the resolution (see introduction). In particular, they found no self-aggregation for runs with horizontal resolutions higher than $2 \mathrm{~km}$ and homogeneous initial conditions. We cannot explain this dependence here but suggest a possible explanation in the conclusions below.

\section{Conclusions}

Self-aggregation of convection can be described as a phase separation process of dry and moist air if the humidity perturbations amplify because of local processes and the spatial coupling is diffusive. As the details of the feedback loop do not affect the pattern formation, this could explain why the appearance of self-aggregation is similar even though the relevant processes have been found to vary. Instead of entering into the question of which mechanism drives self-aggregation, we compare three different time evolution equations for local humidity perturbations introduced by Bretherton et al. (2005), Craig and Mack (2013), and Emanuel et al. (2014) in order to derive a model that explains the spatial evolution of self-aggregation. While differing in details, the homogeneous state in each model is unstable to small perturbations; that is, moist perturbations get moister while dry perturbations get drier.

To determine how humidity perturbations evolve not only in time but also in space, we need to account for the horizontal exchange of humidity. During self-aggregation, convective activity becomes increasingly localized in the moist regions while at the same time humidity increases in regions of increased convective activity. Introducing a stochastic model where convection is more likely to occur 
in moist regions and where convection increases humidity within a finite region around the cloud center, we show that this is approximated by a reaction-diffusion equation: a strictly local increase of humidity due to the number of clouds at that location and a diffusion term.

Including the complete positive feedback loops introduced by Bretherton et al. (2005), Craig and Mack (2013), and Emanuel et al. (2014) in the reactiondiffusion equation, instead of only accounting for the moistening of the atmosphere due to increased convection, shows that these three models are described by time-dependent Ginzburg-Landau equations, a specific type of reaction-diffusion equation that describes phase separation by a process called coarsening, first applied to self-aggregation by Craig and Mack (2013). During coarsening an initially well-mixed state separates into small regions of the two stable phases that then grow in time. The two phases here correspond to the moist and dry regions. This generic evolution is observed in the spatial evolution of all three models and accords well with the appearance of self-aggregation observed in numerical simulations. It is interesting to note that the drying and moistening rates of the three models differ, a property that also differs in numerical studies. Apart from this qualitative description, coarsening has some universal properties that are independent of the mechanisms driving self-aggregation. Most importantly, the upscale growth minimizes the length of the boundary between moist and dry regions and follows a power law with an exponent of $1 / 2$.

Comparison of these universal properties with properties of RCE simulations observed in other studies suggests that coarsening might well describe the early stages of self-aggregation. In particular, we find that the upscale growth in RCE simulations performed by Hohenegger and Stevens (2016) approximately follows the expected scaling relation from about day 1 up to at least day 15 of the simulations if we exclude the first day as a triggering phase. The presence of a triggering phase, also apparent in other RCE simulations showing self-aggregation, cannot be explained by the theoretical models introduced here. A plausible explanation, however, is that a large enough fluctuation in the humidity content has to be present for self-aggregation to start. This could also explain why self-aggregation, in case of homogeneous initial conditions, starts more readily for coarse resolutions, as a coarse resolution makes the formation of large perturbations more likely. In addition to the upscale growth, we show that the tendency of channel-like domains to form banded moist structures while square domains result in circular moist regions as well as the domain-size dependence of self-aggregation can be explained by coarsening.
The findings in this paper emphasize three important points. First, the similarity in the appearance of selfaggregation in different numerical simulations does not mean that there is a unique mechanism but rather that they have the same underlying pattern formation process. Second, to understand which pattern formation process is relevant, we have to understand how humidity is exchanged horizontally. We suggest here that the observed pattern formation is, at least in the early stages, due to coarsening. Coarsening applies if the spatial exchange of humidity is diffusive but differs in the case of strong advection, which has been found to become important at later times, at least in the study by Wing and Emanuel (2014). Third, if, at least initially, the respective pattern formation process is described by coarsening, the spatial evolution of self-aggregation (even when driven by different mechanisms) initially resembles the spatial evolution of other systems exhibiting coarsening (e.g., the Ising model) and shares the same universal properties.

Even though RCE simulations represent the base state of the tropical atmosphere, their setup is highly idealized, and so the question arises of how important self-aggregation, and thus also coarsening, is under more-realistic conditions. One key motivation to study self-aggregation is that it suggests a link between convective organization and climate sensitivity (e.g., Bony et al. 2015). In particular, it has been argued that selfaggregation can enhance or oppose changes in sea surface temperature driven by other processes (e.g., Khairoutdinov and Emanuel 2010). To assess whether self-aggregation leads to a robust response in different models is one of the main questions addressed in the ongoing Radiative-Convective Equilibrium Model Intercomparison Project (Wing et al. 2018). The reason why self-aggregation impacts climate sensitivity is because it impacts the atmospheric mean state; for example, the domain mean humidity decreases with increasing degree of organization (e.g., Bretherton et al. 2005). These results have triggered observational studies that have confirmed that the atmospheric mean state changes systematically with the degree of organization. In particular, Tobin et al. (2012) used observations to show that the mean atmospheric humidity is lower in regions in which convection is more organized. A comprehensive review of convective organization in observations and the relation to self-aggregation is given in Holloway et al. (2017). While the models introduced here do not make any predictions about how the climate sensitivity changes with changing SST, it is worthwhile to note that coarsening only occurs if the humidity content is locally bistable (Craig and Mack 2013). As the atmosphere might be bistable only for a range of SSTs (Emanuel et al. 2014), 
coarsening can readily explain the transition from an unaggregated to an aggregated state with changing SST.

Finally, understanding self-aggregation has been suggested to be important for understanding well known forms of convective organization. As has been stated in the introduction, self-aggregation clusters resemble observed types of convective organization (tropical cyclones, Madden-Julian oscillation) if the simulation setup includes the effect of rotation. While rotation thus modifies the properties of the resulting convective organization, the processes leading to self-aggregation are still expected to contribute to the organization. For this reason, understanding SA has been suggested to be important for tropical cyclones (e.g., Nolan et al. 2007) and the Madden-Julian oscillation (e.g., Bretherton et al. 2005). Given that the mechanisms driving selfaggregation are important in more realistic conditions, self-aggregation mechanisms might even impact the tropical rain belts (e.g., Bony et al. 2015). While we note that the models presented here represent SA only in the very idealized nonrotating RCE setup, additional complexity can easily be included; for example, a nonhomogeneous probability of triggering convection could be used to study the implications of the model presented here for the tropical rain belts.

Acknowledgments. This article is based on chapter 4 of the Ph.D. thesis of the first author (Windmiller 2017). We thank Cathy Hohenegger for giving us access to the model output used in section 5 and for helpful comments regarding the presentation of the results. This work was funded by the German Federal Ministry of Education and Research (BMBF) within the framework programme "Research for Sustainable Development (FONA)" (www.fona.de) under 01LK1507D through the High Definition Clouds and Precipitation for Climate Prediction $[\mathrm{HD}(\mathrm{CP}) 2]$ project.

\section{REFERENCES}

Arnold, N. P., and W. M. Putman, 2018: Nonrotating convective self-aggregation in a limited area AGCM. J. Adv. Model. Earth Syst., 10,1029-1046, https://doi.org/10.1002/2017MS001218.

Becker, T., B. Stevens, and C. Hohenegger, 2017: Imprint of the convective parameterization and sea-surface temperature on large-scale convective self-aggregation. J. Adv. Model. Earth Syst., 9, 1488-1505, https://doi.org/10.1002/2016MS000865.

Bony, S., and Coauthors, 2015: Clouds, circulation and climate sensitivity. Nat. Geosci., 8, 261-268, https://doi.org/10.1038/ ngeo2398.

Bray, A. J., 1994: Theory of phase-ordering kinetics. Adv. Phys., 43, 357-459, https://doi.org/10.1080/00018739400101505.

Bretherton, C. S., M. E. Peters, and L. E. Back, 2004: Relationships between water vapor path and precipitation over the tropical oceans. J. Climate, 17, 1517-1528, https://doi.org/10.1175/ 1520-0442(2004)017<1517:RBWVPA > 2.0.CO;2.
, P. N. Blossey, and M. Khairoutdinov, 2005: An energybalance analysis of deep convective self-aggregation above uniform SST. J. Atmos. Sci., 62, 4273-4292, https://doi.org/ 10.1175/JAS3614.1.

Coppin, D., and S. Bony, 2015: Physical mechanisms controlling the initiation of convective self-aggregation in a general circulation model. J. Adv. Model. Earth Syst., 7, 2060-2078, https://doi.org/10.1002/2015MS000571.

Craig, G. C., and J. M. Mack, 2013: A coarsening model for selforganization of tropical convection. J. Geophys. Res. Atmos., 118, 8761-8769, https://doi.org/10.1002/jgrd.50674.

Derbyshire, S., I. Beau, P. Bechtold, J.-Y. Grandpeix, J.-M. Piriou, J.-L. Redelsperger, and P. Soares, 2004: Sensitivity of moist convection to environmental humidity. Quart. J. Roy. Meteor. Soc., 130, 3055-3079, https://doi.org/10.1256/qj.03.130.

Doswell, C. A., 1985: The operational meteorology of convective weather. Volume II: Storm scale analysis. NOAA Tech. Memo., 240 pp.

Einstein, A., 1905: Über die von der molekularkinetischen Theorie der Wärme geforderte Bewegung von in ruhenden Flüssigkeiten suspendierten Teilchen. Ann. Phys., 322, 549-560, https://doi.org/ 10.1002/andp.19053220806.

Emanuel, K., A. A. Wing, and E. M. Vincent, 2014: Radiativeconvective instability. J. Adv. Model. Earth Syst., 6, 75-90, https://doi.org/10.1002/2013MS000270.

Held, I. M., R. S. Hemler, and V. Ramaswamy, 1993: Radiativeconvective equilibrium with explicit two-dimensional moist convection. J. Atmos. Sci., 50, 3909-3927, https://doi.org/ 10.1175/1520-0469(1993)050<3909:RCEWET >2.0.CO;2.

Hohenegger, C., and B. Stevens, 2016: Coupled radiative convective equilibrium simulations with explicit and parameterized convection. J. Adv. Model. Earth Syst., 8, 1468-1482, https:// doi.org/10.1002/2016MS000666.

Holloway, C. E., and S. J. Woolnough, 2016: The sensitivity of convective aggregation to diabatic processes in idealized radiative-convective equilibrium simulations. J. Adv. Model. Earth Syst., 8, 166-195, https://doi.org/10.1002/2015MS000511.

, A. A. Wing, S. Bony, C. Muller, H. Masunaga, T. S. L'Ecuyer, D. D. Turner, and P. Zuidema, 2017: Observing convective aggregation. Surv. Geophys., 38, 1199-1236, https://doi.org/ 10.1007/s10712-017-9419-1.

Houze, R. A., Jr., 2004: Mesoscale convective systems. Rev. Geophys., 42, 1-43, https://doi.org/10.1029/2004RG000150.

Jeevanjee, N., and D. M. Romps, 2013: Convective self-aggregation, cold pools, and domain size. Geophys. Res. Lett., 40, 994-998, https://doi.org/10.1002/grl.50204.

Kempf, A., 2013: Investigation of self-aggregation of tropical convection. Ph.D. thesis, Ludwig-Maximilians Universität München, $111 \mathrm{pp}$.

_ _ 2014: Testing a phase transition theory for convective organisation. Ph.D. thesis, Ludwig-Maximilians Universität München, $109 \mathrm{pp}$.

Khairoutdinov, M., and K. Emanuel, 2010: Aggregated convection and the regulation of tropical climate. 29th Conf. on Hurricanes and Tropical Meteorology, Tucson, AZ, Amer. Meteor. Soc., P2.69, https://ams.confex.com/ams/29Hurricanes/techprogram/ paper_168418.htm.

Krapivsky, P. L., S. Redner, and E. Ben-Naim, 2010: A Kinetic View of Statistical Physics. Cambridge University Press, 488 pp., https://doi.org/10.1017/CBO9780511780516.

Mapes, B., and R. Neale, 2011: Parameterizing convective organization to escape the entrainment dilemma. J. Adv. Model. Earth Syst., 3, M06004, https://doi.org/10.1029/2011MS000042. 
— E. S. Chung, W. M. Hannah, H. Masunaga, A. J. Wimmers, and C. S. Velden, 2018: The meandering margin of the meteorological moist tropics. Geophys. Res. Lett., 45, 1177-1184, https://doi.org/10.1002/2017GL076440.

Muller, C., and I. M. Held, 2012: Detailed investigation of the self-aggregation of convection in cloud-resolving simulations. J. Atmos. Sci., 69, 2551-2565, https://doi.org/10.1175/ JAS-D-11-0257.1.

- , and S. Bony, 2015: What favors convective aggregation and why? Geophys. Res. Lett., 42, 5626-5634, https://doi.org/ 10.1002/2015GL064260.

Nolan, D. S., E. D. Rappin, and K. A. Emanuel, 2007: Tropical cyclogenesis sensitivity to environmental parameters in radiative-convective equilibrium. Quart. J. Roy. Meteor. Soc., 133, 2085-2107, https://doi.org/10.1002/qj.170.

Parsons, D. B., K. Yoneyama, and J.-L. Redelsperger, 2000: The evolution of the tropical western Pacific atmosphere-ocean system following the arrival of a dry intrusion. Quart. J. Roy. Meteor. Soc., 126, 517-548, https://doi.org/10.1002/qj.49712656307.

Peters, O., and J. D. Neelin, 2006: Critical phenomena in atmospheric precipitation. Nat. Phys., 2, 393-396, https://doi.org/ 10.1038/nphys 314 .

Popke, D., B. Stevens, and A. Voigt, 2013: Climate and climate change in a radiative-convective equilibrium version of ECHAM6. J. Adv. Model. Earth Syst., 5, 1-14, https://doi.org/ 10.1029/2012MS000191.

Posselt, D. J., S. van den Heever, G. Stephens, and M. R. Igel, 2012: Changes in the interaction between tropical convection, radiation, and the large-scale circulation in a warming environment. J. Climate, 25, 557-571, https://doi.org/10.1175/2011JCLI4167.1.

Redelsperger, J.-L., D. B. Parsons, and F. Guichard, 2002: Recovery processes and factors limiting cloud-top height following the arrival of a dry intrusion observed during TOGA COARE. J. Atmos. Sci., 59, 2438-2457, https://doi.org/10.1175/ 1520-0469(2002)059<2438:RPAFLC >2.0.CO;2.

Reed, K. A., B. Medeiros, J. T. Bacmeister, P. H. Lauritzen, K. A. Reed, B. Medeiros, J. T. Bacmeister, and P. H. Lauritzen, 2015: Global radiative-convective equilibrium in the Community Atmosphere Model, version 5. J. Atmos. Sci., 72, 2183 2197, https://doi.org/10.1175/JAS-D-14-0268.1.

Seifert, A., and T. Heus, 2013: Large-eddy simulation of organized precipitating trade wind cumulus clouds. Atmos. Chem. Phys., 13, 5631-5645, https://doi.org/10.5194/acp-13-5631-2013.

Sessions, S. L., S. Sugaya, D. J. Raymond, and A. H. Sobel, 2010: Multiple equilibria in a cloud-resolving model using the weak temperature gradient approximation. J. Geophys. Res., 115, D12110, https://doi.org/10.1029/2009JD013376.

, M. J. Herman, and S. Sentić, 2015: Convective response to changes in the thermodynamic environment in idealized weak temperature gradient simulations. J. Adv. Model. Earth Syst., 7, 712-738, https://doi.org/10.1002/2015MS000446.

, S. Sentić, and M. J. Herman, 2016: The role of radiation in organizing convection in weak temperature gradient simulations.
J. Adv. Model. Earth Syst., 8, 244-271, https://doi.org/10.1002/ $2015 \mathrm{MS} 000587$.

Sethna, J. P., 2006: Statistical Mechanics: Entropy, Order Parameters and Complexity. Oxford University Press, $376 \mathrm{pp}$.

Sobel, A. H., G. Bellon, and J. Bacmeister, 2007: Multiple equilibria in a single-column model of the tropical atmosphere. Geophys. Res. Lett., 34, L22804, https://doi.org/10.1029/ 2007GL031320.

Stein, T. H. M., C. E. Holloway, I. Tobin, and S. Bony, 2017: Observed relationships between cloud vertical structure and convective aggregation over tropical ocean. J. Climate, 30, 2187-2207, https://doi.org/10.1175/JCLI-D-16-0125.1.

Tobin, I., S. Bony, and R. Roca, 2012: Observational evidence for relationships between the degree of aggregation of deep convection, water vapor, surface fluxes, and radiation. J. Climate, 25, 6885-6904, https://doi.org/10.1175/ JCLI-D-11-00258.1.

Tompkins, A. M., 2001: Organization of tropical convection in low vertical wind shears: The role of water vapor. J. Atmos. Sci., 58, 529-545, https://doi.org/10.1175/1520-0469(2001) 058<0529:OOTCIL $>2.0$.CO;2.

—_, and G. C. Craig, 1998: Radiative-convective equilibrium in a three-dimensional cloud-ensemble model. Quart. J. Roy. Meteor. Soc., 124, 2073-2097, https://doi.org/10.1002/ qj.49712455013.

Windmiller, J. M., 2017: Organization of tropical convection. Ph.D. thesis, Ludwig-Maximilians-Universität München, 137 pp., http://nbn-resolving.de/urn:nbn:de:bvb:19-212454.

Wing, A. A., and K. A. Emanuel, 2014: Physical mechanisms controlling self-aggregation of convection in idealized numerical modeling simulations. J. Adv. Model. Earth Syst., 6, 59-74, https://doi.org/10.1002/2013MS000269.

— , and T. W. Cronin, 2016: Self-aggregation of convection in long channel geometry. Quart. J. Roy. Meteor. Soc., 142, 1-15, https://doi.org/10.1002/qj.2628.

__, K. Emanuel, C. E. Holloway, and C. Muller, 2017: Convective self-aggregation in numerical simulations: A review. Surv. Geophys., 38, 1173-1197, https://doi.org/10.1007/ s10712-017-9408-4.

— K. K. Aeed, M. Satoh, B. Stevens, S. Bony, and T. Ohno, 2018: Radiative-Convective Equilibrium Model Intercomparison Project. Geosci. Model Dev., 11, 793-813, https:// doi.org/10.5194/gmd-11-793-2018.

Yang, D., 2018a: Boundary layer diabatic processes, the virtual effect, and convective self-aggregation. J. Adv. Model. Earth Syst., 10, 2163-2176, https://doi.org/10.1029/2017MS001261.

_ 2018b: Boundary layer height and buoyancy determine the horizontal scale of convective self-aggregation. J. Atmos. Sci., 75, 469-478, https://doi.org/10.1175/JAS-D-17-0150.1.

Yano, J.-I., C. Liu, and M. W. Moncrieff, 2012: Self-organized criticality and homeostasis in atmospheric convective organization. J. Atmos. Sci., 69, 3449-3462, https://doi.org/10.1175/ JAS-D-12-069.1. 